\title{
Radiative capture of nucleons at astrophysical energies with single-particle states
}

\author{
J.T. Huang ${ }^{\mathrm{a}, 1}$, C. A. Bertulani ${ }^{\mathrm{a}}$, V. Guimarães ${ }^{\mathrm{b}}$ \\ ${ }^{a}$ Department of Physics, Texas A\&M University-Commerce, Commerce, TX 75429, USA \\ ${ }^{b}$ Instituto de Física, Universidade de São Paulo P.O.Box 66318, 05389-970 São Paulo, \\ SP, Brazil
}

\begin{abstract}
Radiative capture of nucleons at energies of astrophysical interest is one of the most important processes for nucleosynthesis. The nucleon capture can occur either by a compound nucleus reaction or by a direct process. The compound reaction cross sections are usually very small, specially for light nuclei. The direct capture proceeds either via the formation of a single-particle resonance, or a non-resonant capture process. In this work we calculate radiative capture cross sections and astrophysical S-factors for nuclei in the mass region $A<20$ using single-particle states. We carefully discuss the parameter fitting procedure adopted in the simplified two-body treatment of the capture process. Then we produce a detailed list of cases for which the model works well. Useful quantities, such as spectroscopic factors and asymptotic normalization coefficients, are obtained and compared to published data.
\end{abstract}

Key words: radiative capture, single-particle model, light nuclei

PACS: 25.40.Lw, 24.50.+g, 26.20.-f

\section{Contents}

1 Introduction 7

2 Direct capture 8

\footnotetext{
${ }^{*}$ Corresponding author

Email addresses: jhuang1@leo.tamu-commerce.edu (J.T. Huang), carlos_bertulani@tamu-commerce.edu (C. A. Bertulani), valdirg@dfn.if.usp.br (V. Guimarães)
} 
2.1 Potentials and Wavefunctions . . . . . . . . . . 8

2.2 Radiative capture cross sections . . . . . . . . . . . . . 10

2.3 Asymptotic normalization coefficients . . . . . . . . . . . 12

3 Proton capture 14

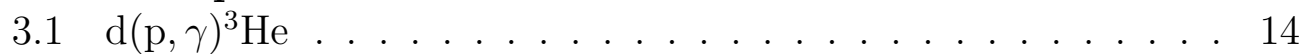

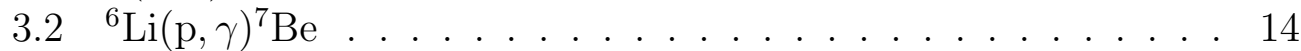

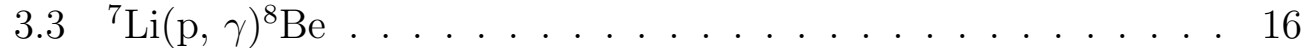

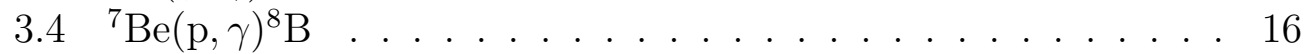

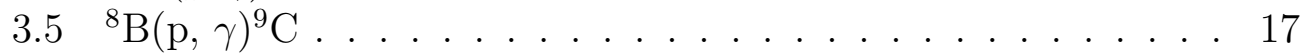

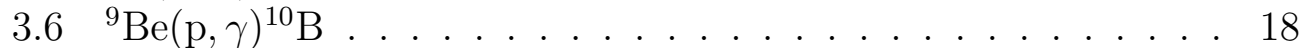

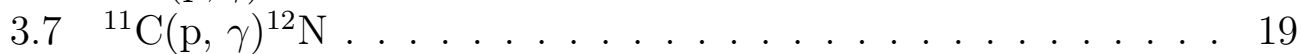

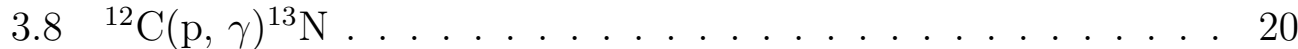

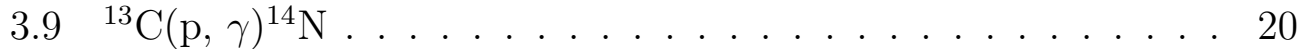

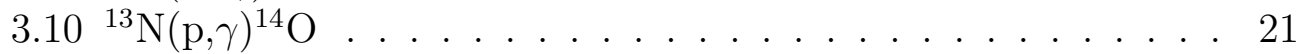

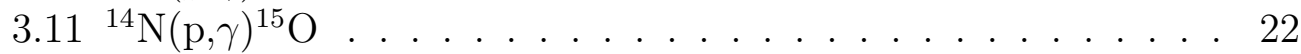

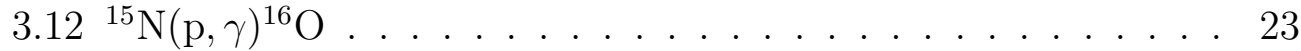

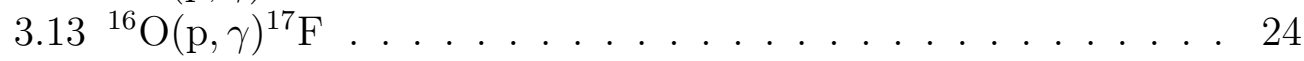

$3.14{ }^{20} \mathrm{Ne}(\mathrm{p}, \gamma)^{21} \mathrm{Na} \ldots \ldots \ldots \ldots \ldots \ldots \ldots . \ldots \ldots \ldots$

4 Neutron capture 25

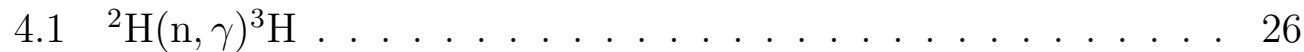

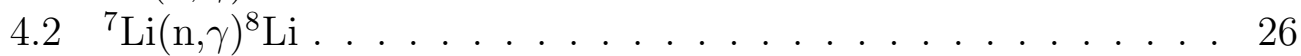

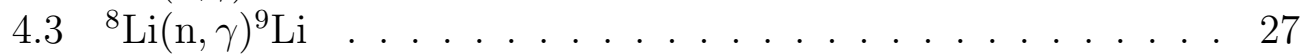

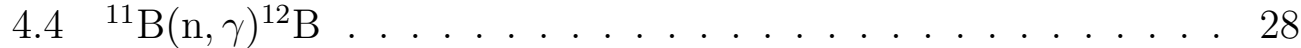

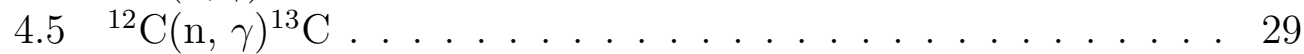

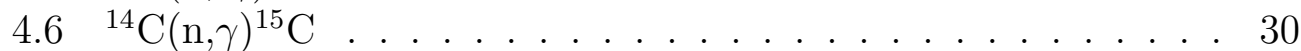

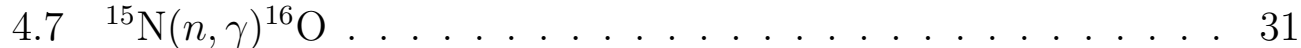

$4.8{ }^{16} \mathrm{O}(n, \gamma)^{17} \mathrm{O} \ldots \ldots \ldots \ldots \ldots \ldots \ldots \ldots \ldots \ldots$

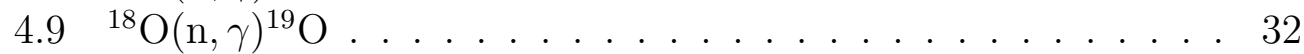

5 Sensitivity on the potential depth parameter 33

6 ANCs from single-particle models 34

7 Final conclusions 


\section{List of Tables}

1 Parameters of the single-particle potentials, except for few cases explicitly mentioned in the text. . . . . . . . . . 43

2 Binding energy $\left(E_{b}\right.$, in $\left.\mathrm{MeV}\right)$, central potential depth of bound state $\left(V_{b}\right.$, in $\left.\mathrm{MeV}\right)$, spectroscopic factor $(S F)$, single-particle asymptotic normalization coefficients $\left(b\right.$, in $\left.\mathrm{fm}^{-1 / 2}\right)$, the factor that multiplies S-factor if the integration in Eq. 6] starts at $r=R_{0}$ (nuclear radius) and S-factor at zero energy $(S(0)$, in $\mathrm{eV}$ b) for radiative proton capture reactions. . . . . . . . 44

3 Binding energy $\left(E_{b}\right.$, in $\left.\mathrm{MeV}\right)$, central potential depth of bound state $\left(V_{b}\right.$, in $\left.\mathrm{MeV}\right)$, spectroscopic factor $(S F)$, single-particle asymptotic normalization coefficients $\left(b\right.$, in $\left.\mathrm{fm}^{-1 / 2}\right)$ and the factor multiplying the S-factor assuming that the integration in Eq. 6] starts at $r=R_{0}$ (nuclear radius). . . . . . . . . . . . 45

4 Cross sections at $0.4 \mathrm{MeV}$ for the capture to the ground state of the reaction ${ }^{16} \mathrm{O}(\mathrm{p}, \gamma){ }^{17} \mathrm{~F}$ with that of ${ }^{16} \mathrm{O}(\mathrm{n}, \gamma){ }^{17} \mathrm{O} . \ldots . .45$

\section{List of Figures}

$1 \mathrm{~d}(p, \gamma)^{3} \mathrm{He} \ldots \ldots \ldots \ldots \ldots$

$2 \quad{ }^{6} \mathrm{Li}(\mathrm{p}, \gamma)^{7} \mathrm{Be} \ldots \ldots \ldots \ldots \ldots$

3 (Color online). Potential model calculation for the reaction ${ }^{7} \mathrm{Li}(\mathrm{p}, \gamma)^{8}$ Be. Experimental data are from Ref. [25]. . . . . . 47

4 (Color online). Single-particle model calculations for the reaction ${ }^{7} \mathrm{Be}(p, \gamma)^{8} \mathrm{~B}$. The dashed-dotted line is the calculation for the M1 resonance at $E_{c m}=0.63 \mathrm{MeV}$ and the dotted line is for the non-resonant capture. Experimental data are from Refs. [29, 30, 31, 28, 32, 33]. The total S factor is shown as a solid line. . . . . . . . . . . . . . 47

5 (Color online). Single-particle model calculations for the reaction ${ }^{8} \mathrm{~B}(\mathrm{p}, \gamma)^{9} \mathrm{C}$ (solid line). The open circle at $E=0$ is from Refs. [41, 42]. The result from Ref. [40] $\left(\lambda_{\text {scatt }}=0.55 \mathrm{fm}\right)$ is shown as a dashed line. . . . . . . . . . . . . 48 
6 (Color online). Single-particle model calculations for the reaction ${ }^{9} \mathrm{Be}(\mathrm{p}, \gamma){ }^{10} \mathrm{~B}$ (solid line). The experimental data are from Ref. [48]. The fits to the resonances, done in Ref. [48], are shown as dashed lines. DC results from Ref. [49] and Ref. [48] are shown as a dotted-dashed line and a dotted line, respectively. The curve passing through the experimental data points is the sum of our DC calculation and the resonance fits, given by the dashed lines. . . . . . . . . . . . . . . . . 48

7 (Color online). Single-particle model calculations for the reaction ${ }^{11} \mathrm{C}(\mathrm{p}, \gamma){ }^{12} \mathrm{~N}$ (solid line). R-matrix results from Ref. [53] are also shown by dashed lines (resonances) and a dotted line (non-resonant). . . . . . . . . . . . . . . 49

8 (Color online). Single-particle model calculations for the reaction ${ }^{12} \mathrm{C}(\mathrm{p}, \gamma){ }^{13} \mathrm{~N}$ are shown as a dashed line (DC), a dotted line ( $E 1$ resonance) and a solid line (total). The experimental data are from Ref. [58]. The potential model results from Ref. [57] are shown as dotted-dashed lines. . . . . . . . . . . . . 49

9 (Color online). Single-particle model calculations for the reaction ${ }^{13} \mathrm{C}(\mathrm{p}, \gamma){ }^{14} \mathrm{~N}$ are shown as a dashed line (DC), a dotted line ( $E 1$ resonance) and a solid line (total). The experimental data are from Ref. [66]. The potential model results from Ref. 57] are shown as dotted-dashed lines. . . . . . . . . . . 50

10 (Color online). Single-particle model calculations for the reaction ${ }^{13} \mathrm{~N}(\mathrm{p}, \gamma){ }^{14} \mathrm{O}$ are shown as a dotted-dashed line (nonresonant) and a solid line (E1 resonance). R-matrix results from Ref. [68] are also shown as a dashed line (resonance) and a dotted line (non-resonant). . . . . . . . . . . . 50

11 (Color online). Single-particle model calculations for ${ }^{14} \mathrm{~N}(\mathrm{p}, \gamma)^{15} \mathrm{O}$ capture to the $6.793 \mathrm{MeV}$ excited state of ${ }^{15} \mathrm{O}$. Dashed line is for the non-resonat capture, dotted line is for the M1 resonance, and the solid line is the total S-factor. The experimental data are from Refs. [78, 82, 83]. The dotted-dashed line is a R-matrix fit obtained in Ref. [78] with the channel radius $a=5.5 \mathrm{fm}$ (this curve is almost invisible because it is very close to our results). . . . . . . . . . . . . . . . 51 
12 (Color online). Single-particle model calculation for the reaction ${ }^{15} \mathrm{~N}(\mathrm{p}, \gamma){ }^{16} \mathrm{O}$ (solid line). The experimental data are from Refs. [86, 91]. Dashed lines are Breit-Wigner fits to the resonances, as described in Ref. [86]. The dotted line is a non-resonant capture of Ref. [86]. The dotted-dashed line represents the non-resonant capture calculation from Ref. [88]. 51

13 (Color online). Single-particle model calculation for the reaction ${ }^{16} \mathrm{O}(\mathrm{p}, \gamma){ }^{17} \mathrm{~F}$. The dotted line and the dashed line are for the capture to the ground state and to the first excited state respectively. The experimental data are from Refs. 94, 95, 93, 96]. The dotted-dashed lines are the result of shell model calculations published in Ref. [97]. . . . . . . . . . . . 52

14 (Color online). Single-particle model calculation for the reaction ${ }^{20} \mathrm{Ne}(\mathrm{p}, \gamma){ }^{21} \mathrm{Na}$. Upper solid line is for the capture to the $2.425 \mathrm{MeV}$ excited state of ${ }^{20} \mathrm{Ne}$ and lower solid line for the $0.332 \mathrm{MeV}$ excited state. Experimental data are from Ref. [100]. The dashed and dotted lines are theoretical results from Ref. [100] and Ref. [101], respectively . . . . . . . . . . . 5 52

15 (Color online). Single-particle model calculation for ${ }^{2} \mathrm{H}(n, \gamma)^{3} \mathrm{H}$ (solid line). The experimental data are from Ref. [110]. The phenomenological results (parameter fit) from from Ref. [110] are shown by dashed and dotted lines. Also shown are microscopic calculations with (open trianges) and without (solid triangles) a three-body interaction. . . . . . . . . . . . 53

16 (Color online). Single-particle model calculation for the reaction ${ }^{7} \operatorname{Li}(\mathrm{n}, \gamma){ }^{8} \mathrm{Li}$. The dashed and dotted lines are for the capture to the ground state and first excited state, respectively. The dotted-dashed line is the calculated M1 resonance. The total cross section is shown as a solid line. The calculation result from Ref. [115] is shown as a dotted-dotted-dashed line. The experimental data are from refs. [115, 116, 117, 118, 119]. 53

17 (Color online). Single-particle model calculation for ${ }^{8} \mathrm{Li}(n, \gamma)^{9} \mathrm{Li}$. The solid and the dashed lines are the calculations for the capture to the ground and the 1st excited states, respectively. The experimental data are from Ref. [127] using the Coulomb dissociation of ${ }^{9} \mathrm{Li}$ on $\mathrm{Pb}$ targets at $28.5 \mathrm{MeV} / \mathrm{A}$ beam energy. The dotted line is the calculation reported in Ref. [128] for the capture to the ground state. . . . . . . . . . . . 54 
18 (Color online). Single-particle model calculation for the (nonresonant) capture reaction ${ }^{11} \mathrm{~B}(n, \gamma){ }^{12} \mathrm{~B}$ (solid line). The experimental data are from Ref. [131]. The dashed line is a sum of fitted Breit-Wigners superimposed to the non-resonant capture calculation, following Ref. [131]. . . . . . . . . . . 54

19 (Color online). Single-particle model calculation for ${ }^{12} \mathrm{C}(\mathrm{n}$, $\gamma{ }^{13} \mathrm{C}$ (solid line). The upper panel is for the capture to the ground state whereas the lower one is for capture to the 2nd excited state. The experimental data are from Ref. 134] (filled square) and Ref. [135] (filled triangle). The theoretical results from Ref. [135] and Ref. [136] are shown by the dashed and the dotted lines, respectively. . . . . . . . . . . 5 55

20 (Color online). The same as Fig. 19, but for the transitions to the 1st excited state (upper panel) and to the 3rd excited state (lower panel). . . . . . . . . . . . . . . . . 5 56

21 (Color online). Single-particle model calculation for the reaction ${ }^{14} \mathrm{C}(n, \gamma){ }^{15} \mathrm{C}$ (solid line). The experimental data are from Ref. [142]. The dashed line is the result from Ref. [143] using a similar potential model. . . . . . . . . . . . . . . . 57

22 (Color online). Single-particle model calculation results for ${ }^{15} \mathrm{~N}(n, \gamma){ }^{16} \mathrm{~N}$ (solid line). The experimental data are from Ref. [145]. The non-resonant capture calculations of Ref. [145] is shown by open circles. Increasing the values of the spectroscopic values by $30 \%$ (compatible with the experimental errors) yields the dashed line. . . . . . . . . . . . . . . . 57

23 (Color online). Single-particle model calculation for reaction ${ }^{16} \mathrm{O}(n, \gamma)^{17} \mathrm{O}$ (solid lines). The experimental data are from Ref. [148]. Top panel: the capture to the ground state (dotted line, filled circles) and first excited state (dashed line, filled triangles) of ${ }^{17} \mathrm{O}$ are shown separately. The results of a microscopic multicluster model from Ref. [149] are shown by dotted-dashed lines for comparison. Bottom panel: the total cross section of ${ }^{18} \mathrm{O}(n, \gamma){ }^{19} \mathrm{O}$ (solid line). The result from Ref. [150] is shown as a dashed line. . . . . . . . . . . . . 58 
24 (Color online). Single-particle model calculation for the reaction of ${ }^{18} \mathrm{O}(n, \gamma){ }^{19} \mathrm{O}$ (solid line). The experimental data are from Ref. [151]. The non-resonant capture calculation from Ref. [151] and [147] are shown as open circles and dashed line, respectively.

25 (Color online). Ratio between the S-factor at $E=0$ calculated with a potential depth $V_{c}$ and the S-factor calculated with a zero potential depth: $S\left(0, V_{c}\right) / S(0,0)$. The open circle corresponds to the value of $V_{c}$ used in the calculation presented in figure 12. . . . . . . . . . . . . . . . . 59

26 (Color online). Our ANCs $\left(\sqrt{(S F) b^{2}}\right)$ divided by the ANCs obtained from references mentioned in the text as function of the mass number $A$. The solid circles are for proton capture whereas the solid triangles are for neutron capture. The dashed line is equal to unity. . . . . . . . . . . . . . . .60

\section{Introduction}

Fusion reactions relevant for astrophysics proceed via compound-nucleus formation, with a very large number of resonances involved, or by direct capture, with only few or no resonances. To calculate direct capture cross sections one needs to solve the many body problem for the bound and continuum states of relevance for the capture process (for a review see, [1]). A much simpler, and popular, solution is based on a potential model to obtain single-particle energies and wavefunctions [2]. The model assumes two structureless particles interacting via a potential with a relative coordinate dependence determined by a set of adjusting parameters. Often, this solution is good enough to yield cross sections within the accuracy required to reproduce the experiments.

In this article we explore the single-particle model to perform a systematic study of radiative capture reactions for several light nuclei. This study has not yet been reported in the literature, where one finds its application to isolated cases. It is also useful to obtain potential parameters for other reaction channels and predict quantities of interest, such as spectroscopic factors (SF) and asymptotic normalization coefficients (ANC).

This paper is organized as follows. In section II we summarize the theoretical tools used in the single-particle description of direct capture (DC) reactions. We show how potentials and wavefunctions are built, followed 
by a description of how radiative capture cross sections are obtained. Then we discuss the derivation and interpretation of the asymptotic normalization coefficients. In section III we present and discuss the results for radiative proton capture, whereas in section IV we present and discuss the results for radiative neutron capture. The sensitivity of the S-factors on the potential parameters is discussed in section V. A summary of the ANCs obtained in this work is described in section VI. Our final conclusions are given in section VII.

\section{Direct capture}

\subsection{Potentials and Wavefunctions}

In this work we adopt nuclear potentials of the form

$$
V(\mathbf{r})=V_{0}(r)+V_{S}(r)(\mathbf{l} . \mathbf{s})+V_{C}(r)
$$

where $V_{0}(r)$ and $V_{S}(r)$ are the central and spin-orbit interactions, respectively, and $V_{C}(r)$ is the Coulomb potential of a uniform distribution of charges:

$$
\begin{aligned}
V_{C}(r) & =\frac{Z_{a} Z_{b} e^{2}}{r} \quad \text { for } \quad r>R_{C} \\
& =\frac{Z_{a} Z_{b} e^{2}}{2 R_{C}}\left(3-\frac{r^{2}}{R_{C}^{2}}\right) \quad \text { for } \quad r<R_{C},
\end{aligned}
$$

where $Z_{i}$ is the charge number of nucleus $i=a, b$.

Here we use a Woods-Saxon (WS) parameterization to build up the potentials $V_{0}(r)$ and $V_{S}(r)$, given by

$$
\begin{aligned}
V_{0}(r) & =V_{0} f_{0}(r), \\
V_{S}(r) & =-V_{S 0}\left(\frac{\hbar}{m_{\pi} c}\right)^{2} \frac{1}{r} \frac{d}{d r} f_{S}(r) \\
\text { with } \quad f_{i}(r) & =\left[1+\exp \left(\frac{r-R_{i}}{a_{i}}\right)\right]^{-1} .
\end{aligned}
$$

The spin-orbit interaction in Eq. 3 is written in terms of the pion Compton wavelength, $\hbar / m_{\pi} c=1.414 \mathrm{fm}$. The parameters $V_{0}, V_{S 0}, R_{0}, a_{0}, R_{S 0}$, and $a_{S 0}$ are chosen to reproduce the ground state energy $E_{B}$ (or the energy of 
an excited state). For this purpose, we define typical values (Table I) for $V_{S 0}, R_{0}, a_{0}, R_{S 0}$, and vary only the depth of the central potential, $V_{0}$. As we discuss later, a different set of potential depths might be used for continuum states.

For neutron and proton capture reactions, there is no need for using another form for the potentials. The WS set of parameters are well suited to describe any reaction of interest, except perhaps for those cases in which one of the partners is a neutron-rich halo nucleus. Then the extended radial dependence leads to unusual forms for the potentials. Also, for capture reactions in which the light partner is either a deuteron, tritium, $\alpha$-particle or a heavier nucleus, folding models are more appropriate. Folding models are based on an effective nucleon-nucleon interaction and nuclear densities which are either obtained experimentally (not really, because only charge densities can be accurately determined from electron-scattering), or calculated from some microscopic model (typically Hartree-Fock or relativistic mean field models). The effective interactions as well as the nuclear densities are subject of intensive theoretical studies, which is beyond the scope of this work. We will restrict our studies to neutron and proton radiative capture reactions based on a nucleon-nucleus interaction of the form of Eq. 1,

The wavefunctions for the nucleon $(\mathrm{n})+$ nucleus $(\mathrm{x})$ system are calculated by solving the radial Schrödinger equation

$$
-\frac{\hbar^{2}}{2 m_{n x}}\left[\frac{d^{2}}{d r^{2}}-\frac{l(l+1)}{r^{2}}\right] u_{\alpha}(r)+V(r) u_{\alpha}(r)=E_{\alpha} u_{\alpha}(r) .
$$

The nucleon $n$, the nucleus $x$, and the $n+x=a$-system have intrinsic spins labeled by $s=1 / 2, I_{x}$ and $J$, respectively. The orbital angular momentum for the relative motion of $n+x$ is described by $l$. It is convenient to couple angular momenta as $\mathbf{l}+\mathbf{s}=\mathbf{j}$ and $\mathbf{j}+\mathbf{I}_{x}=\mathbf{J}$, where $\mathbf{J}$ is called the channel spin. In Eq. 1 for $V$ we use $\mathbf{s . l}=[j(j+1)-l(l+1)-3 / 4] / 2$ and $\alpha$ in Eq. 4 denotes the set of quantum numbers, $\alpha_{b}=\left\{E_{b}, l_{b}, j_{b}, J_{b}\right\}$ for the bound state, and $\alpha_{c}=\left\{E_{c}, l_{c}, j_{c}, J_{c}\right\}$ for the continuum states.

The bound-state wavefunctions are normalized to unity, $\int d r\left|u_{\alpha_{b}}(r)\right|^{2}=$ 1 , whereas the continuum wavefunctions have boundary conditions at infinity given by

$$
u_{\alpha_{c}}(r \rightarrow \infty)=i \sqrt{\frac{m_{n x}}{2 \pi k \hbar^{2}}}\left[H_{l}^{(-)}(r)-S_{\alpha_{c}} H_{l}^{(+)}(r)\right] e^{i \sigma_{l}(E)}
$$


where $S_{\alpha_{c}}=\exp \left[2 i \delta_{\alpha_{c}}(E)\right]$, with $\delta_{\alpha_{c}}(E)$ and $\sigma_{l}(E)$ being the nuclear and the Coulomb phase-shifts, respectively. In Eq. 5, $H_{l}^{( \pm)}(r)=G_{l}(r) \pm i F_{l}(r)$, where $F_{l}$ and $G_{l}$ are the regular and irregular Coulomb wavefunctions. For neutrons the Coulomb functions reduce to the usual spherical Bessel functions, $j_{l}(r)$ and $n_{l}(r)$. With these definitions, the continuum wavefunctions are normalized as $\left\langle u_{E_{c}^{\prime}} \mid u_{E_{c}}\right\rangle=\delta\left(E_{c}^{\prime}-E_{c}\right) \delta_{\alpha \alpha^{\prime}}$.

\subsection{Radiative capture cross sections}

The radiative capture cross sections for $n+x \rightarrow a+\gamma$ and $\pi L(\pi=$ $E,(M)=$ electric (magnetic) L-pole) transitions are calculated with

$$
\begin{aligned}
\sigma_{E L, J_{b}}^{\text {d.c. }} & =\frac{(2 \pi)^{3}}{k^{2}}\left(\frac{E_{n x}+E_{b}}{\hbar c}\right)^{2 L+1} \frac{2\left(2 I_{a}+1\right)}{\left(2 I_{n}+1\right)\left(2 I_{x}+1\right)} \\
& \times \frac{L+1}{L[(2 L+1) ! !]^{2}} \sum_{J_{c} j_{c} l_{c}}\left(2 J_{c}+1\right) \\
& \times\left\{\begin{array}{ccc}
j_{c} & J_{c} & I_{x} \\
J_{b} & j_{b} & L
\end{array}\right\}^{2}\left|\left\langle l_{c} j_{c}\left\|\mathcal{O}_{\pi L}\right\| l_{b} j_{b}\right\rangle\right|^{2},
\end{aligned}
$$

where $E_{b}$ is the binding energy and $\left\langle l_{c} j_{c}\left\|\mathcal{O}_{\pi L}\right\| l_{b} j_{b}\right\rangle$ is the multipole matrix

element. For the electric multipole transitions we have

$$
\begin{aligned}
\left\langle l_{c} j_{c}\left\|\mathcal{O}_{E L}\right\| l_{b} j_{b}\right\rangle & =(-1)^{l_{b}+l_{c}-j_{c}+L-1 / 2} \frac{e_{L}}{\sqrt{4 \pi}} \\
& \times \sqrt{(2 L+1)\left(2 j_{b}+1\right)}\left(\begin{array}{ccc}
j_{b} & L & j_{c} \\
1 / 2 & 0 & -1 / 2
\end{array}\right) \\
& \times \int_{0}^{\infty} d r r^{L} u_{b}(r) u_{c}(r),
\end{aligned}
$$

where $e_{L}$ is the effective charge, which takes into account the displacement of the center-of-mass,

$$
e_{L}=Z_{n} e\left(-\frac{m_{n}}{m_{a}}\right)^{L}+Z_{x} e\left(\frac{m_{x}}{m_{a}}\right)^{L} .
$$

In comparison with the electric dipole transitions the cross sections for magnetic dipole transitions are reduced by a factor of $v^{2} / c^{2}$, where $v$ is the relative velocity of the $n+x$ system. At very low energies, $v \ll c, M 1$ transitions will be much smaller than the electric transitions. Only in the 
case of sharp resonances, the M1 transitions play a significant role, e.g. for the $J=1^{+}$state in ${ }^{8} \mathrm{~B}$ at $E_{R}=630 \mathrm{keV}$ above the proton separation threshold [5, 3]. In general, the potential model is not good to reproduce M1 transition amplitudes [4]. We will explore few situations in which the model works well.

The radiative capture cross sections for $n+x \rightarrow a+\gamma$ and $M 1$ transitions are calculated with

$$
\begin{aligned}
& \left\langle l_{c} j_{c}\left\|\mathcal{O}_{M 1}\right\| l_{b} j_{b}\right\rangle=(-1)^{j_{c}+I_{x}+J_{b}+1} \sqrt{\frac{3}{4 \pi}} \mu_{N} \\
& \times\left\{\frac { 1 } { \widehat { l } _ { b } } e _ { M } \left[\frac{2 \tilde{j}_{b}}{\widehat{l}_{b}}\left(l_{b} \delta_{j_{b}, l_{b}+1 / 2}+\left(l_{b}+1\right) \delta_{j_{b}, l_{b}-1 / 2}\right)\right.\right. \\
& \left.+(-1)^{l_{b}+1 / 2-j_{c}} \frac{\widehat{j}_{b}}{\sqrt{2}} \delta_{j_{b}}, l_{b} \pm 1 / 2 \delta_{j_{c}}, l_{b} \mp 1 / 2\right] \\
& +g_{N} \frac{1}{\widehat{l}_{b}^{2}}\left[(-1)^{l_{b}+1 / 2-j_{b}} \widetilde{j}_{b} \delta_{j_{c}, j_{b}}\right. \\
& \left.-(-1)^{l_{b}+1 / 2-j_{c}} \frac{\widehat{j_{b}}}{\sqrt{2}} \delta_{j_{b}, l_{b} \pm 1 / 2} \delta_{j_{c}, l_{b} \mp 1 / 2}\right] \\
& \left.+g_{x}(-1)^{I_{x}+j_{b}+J_{c}+1} \widehat{J}_{b} \widehat{J}_{c} \widehat{I}_{x} \widetilde{I}_{x}\left\{\begin{array}{ccc}
I_{x} & J_{c} & j_{b} \\
J_{b} & I_{x} & 1
\end{array}\right\}\right\} \\
& \times \int_{0}^{\infty} d r r u_{c}(r) u_{b}(r)
\end{aligned}
$$

where $\tilde{k}=\sqrt{k(k+1)}$ and $\hat{k}=\sqrt{2 k+1}$. The spin g-factor is $g_{N}=5.586$ for the proton and $g_{N}=-3.826$ for the neutron. The magnetic moment of the core nucleus is given by $\mu_{x}=g_{x} \mu_{N}$. If $l_{c} \neq l_{b}$ the magnetic dipole matrix element is zero.

The total direct capture cross section is obtained by adding all multipolarities and final spins of the bound state $\left(E \equiv E_{n x}\right)$,

$$
\sigma^{\text {d.c. }}(E)=\sum_{L, J_{b}}(S F)_{J_{b}} \sigma_{L, J_{b}}^{\text {d.c. }}(E)
$$

where $(S F)_{J_{b}}$ are spectroscopic factors.

For charged particles the astrophysical S-factor for the direct capture from 
a continuum state to the bound state is defined as

$$
\begin{aligned}
& S(E)=E \sigma^{\text {d.c. }}(E) \exp [2 \pi \eta(E)], \\
& \text { with } \quad \eta(E)=Z_{a} Z_{b} e^{2} / \hbar v,
\end{aligned}
$$

where $v$ is the initial relative velocity between $n$ and $x$.

For some resonances, not reproducible with the single-particle model, we will use a simple Breit-Wigner shape parametrization

$$
\sigma_{B W}=\frac{\Gamma}{2 \pi} \frac{\sigma_{0}(E)}{\left(E-E_{R}\right)^{2}+\Gamma^{2} / 4},
$$

where $E_{R}$ is the resonance energy. The function $\sigma_{0}(E)$ is given by

$$
\sigma_{0}(E)=\frac{\pi \hbar^{2}}{2 m_{x n} E} \frac{2 J_{R}+1}{(2 J x+1)\left(2 J_{n}+1\right)} \frac{\Gamma_{n}(E) \Gamma_{\gamma}(E)}{\Gamma(E)}
$$

where the total width $\Gamma=\Gamma_{n}+\Gamma_{\gamma}$ is the sum of the nucleon-decay and the $\gamma$-decay widths. For simplicity, and for the cases treated here, we will assume that the resonances are narrow so that $\sigma_{0}=\sigma\left(E_{R}\right)$.

\subsection{Asymptotic normalization coefficients}

Although the potential model works well for many nuclear reactions of interest in astrophysics, it is often necessary to pursue a more microscopic approach [6, 7] to reproduce experimental data. In a microscopic approach, instead of the single-particle wavefunctions one often makes use of overlap integrals, $I_{b}(\mathbf{r})$, and a many-body wavefunction for the relative motion, $\Psi_{c}(\mathbf{r})$. Both $I_{b}(\mathbf{r})$ and $\Psi_{c}(\mathbf{r})$ might be very complicated to calculate, depending on how elaborated the microscopic model is. The variable $\mathbf{r}$ is the relative coordinate between the nucleon and the nucleus $x$, with all the intrinsic coordinates of the nucleons in $x$ being integrated out. The direct capture cross sections are obtained from the calculation of $\sigma_{L, J_{b}}^{\text {d.c. }} \propto\left|\left\langle I_{b}(r)|| r^{L} Y_{L}|| \Psi_{c}(r)\right\rangle\right|^{2}$.

The imprints of many-body effects will eventually disappear at large distances between the nucleon and the nucleus. One thus expects that the overlap function asymptotically matches the solution of the Schrödinger equation 4. with $V=V_{C}$ for protons and $V=0$ for neutrons. That is, when $r \rightarrow \infty$,

$$
\begin{aligned}
& I_{b}(r)=C_{1} \frac{W_{-\eta, l_{b}+1 / 2}(2 \kappa r)}{r}, \text { for protons } \\
& =C_{2} \sqrt{\frac{2 \kappa}{r}} K_{l_{b}+1 / 2}(\kappa r), \text { for neutrons }
\end{aligned}
$$


where the binding energy of the $n+x$ system is related to $\kappa$ by means of $E_{b}=\hbar^{2} \kappa^{2} / 2 m_{n x}, W_{p, q}$ is the Whittaker function and $K_{\mu}$ is the modified Bessel function. In Eq. 14, $C_{i}$ is the asymptotic normalization coefficient (ANC).

In the calculation of $\sigma_{L, J_{b}}^{\text {d.c. }}$ above, one often meets the situation in which only the asymptotic part of $I_{b}(r)$ and $\Psi_{c}(r)$ contributes significantly to the integral over $r$. In these situations, $\Psi_{c}(r)$ is also well described by a simple two-body scattering wave (e.g. Coulomb waves). Therefore the radial integration in $\sigma_{L, J_{b}}^{\text {d.c }}$ can be done accurately and the only remaining information from the many-body physics at short-distances is contained in the asymptotic normalization coefficient $C_{i}$, i.e. $\sigma_{L, J_{b}}^{\text {d.c. }} \propto C_{i}^{2}$. We thus run into an effective theory for radiative capture cross sections, in which the constants $C_{i}$ carry all the information about the short-distance physics, where the many-body aspects are relevant. It is worthwhile to mention that these arguments are reasonable for proton capture at very low energies, because of the Coulomb barrier.

The spectroscopic factors, $S F$, are usually obtained by adjusting the calculated cross sections to reproduce the experimental ones. Here we try to follow the literature as closely as possible. When experimental data are not available, we use spectroscopic factors taken from the literature. For the cases in which experimental data exist, we also try to use spectroscopic factors published in the literature, and fit the data by varying the depth of the WS potential for the continuum states.

The asymptotic normalization coefficients, $C_{\alpha}$, can also be obtained from the analysis of peripheral, transfer and breakup, reactions. As the overlap integral, Eq. 14, asymptotically becomes a Whittaker function, so does the single particle bound-state wavefunction $u_{\alpha}$, calculated with Eq. 4. If we call the single particle ANC by $b_{i}$, then the relation between the ANC obtained from experiment, or a microscopic model, with the single particle ANC is given by $(S F)_{i} b_{i}^{2}=C_{i}^{2}$. This becomes clear from Eq. 10. The values of $(S F)_{i}$ and $b_{i}$ obtained with the simple potential model are useful telltales of the complex short-range many-body physics of radiative capture reactions. One can also invert this argumentation and obtain spectroscopic factors if the $C_{i}$ are deduced from a many-body model, or from experiment, and the $b_{i}$ are calculated from a single particle potential model [8]. 


\section{Proton capture}

Table 2 summarizes the potential parameters used in cases where the potential model works reasonably well for radiative proton capture reactions. A discussion is presented case by case in the following subsections. Unless otherwise stated, we use the parameters according to Table 1 for the singleparticle potential. The parameters for the continuum potential, $V_{c}$, are the same as for the bound state potential, except for few cases discussed explicitly in the text.

\section{1. $d(p, \gamma)^{3} \mathrm{He}$}

Understanding of the nature of ${ }^{3} \mathrm{He}$, the only stable 3 -body nucleus, constitutes a major advance towards the solution of the general problem of nuclear forces. In particular, it involves the influence of the third nucleon on the interaction between the other two. This latter interaction has been studied extensively in deuteron and in nucleon-nucleon scattering. These are issues beyond the scope of this article. But we will show that a rather good reproduction of the experimental data for the capture reaction $\mathrm{d}(\mathrm{p}, \gamma)^{3} \mathrm{He}$ can be obtained with the simple potential model described in the previous sections.

The $J_{b}=1 / 2^{+}$ground state of ${ }^{3} \mathrm{He}$ is described as a $j_{b}=s_{1 / 2}$ proton coupled to the deuterium core, which has an intrinsic spin $I_{x}=1^{+}$. The gamma-ray transition is dominated by the $E 1$ multipolarity and by incoming $p$ waves. Our results require a spectroscopic factor $S F=0.7$ to fit the experimental data shown in Fig. 1. If we add d-waves to the ground-state there is a negligible change in this value. Thus, the contribution of d-waves in the ground state has been neglected. The experimental data are from Ref. [9] (filled squares), Ref. [10] (open squares), Ref. [11] (open circles), Ref. [12] (filled triangles).

In Ref. [13], the ANC for this reaction was found by an analysis of swave pd and nd scattering. The ANC for the $l=0$ channel was found to be $1.97 \mathrm{fm}^{-1 / 2}\left(C^{2}=3.9 \pm 0.06 \mathrm{fm}^{-1}\right)$ [13]. Our ANC value is $\sqrt{(S F) b^{2}}=$ $1.56 \mathrm{fm}^{-1 / 2}$, which is in good agreement with the more complicated analysis presented in Ref. [13].

\section{2. $\left.{ }^{6} \mathrm{Li}(p, \gamma)\right)^{7} \mathrm{Be}$}

Unlike ${ }^{7} \mathrm{Li},{ }^{6} \mathrm{Li}$ is predicted to be formed at a very low level in Big Bang nucleosynthesis, ${ }^{6} \mathrm{Li} / \mathrm{H}=10^{-14}[14,15]$. Whereas most elements are produced by stellar nucleosynthesis, lithium is mainly destroyed in stellar interiors by 
thermonuclear reactions with protons. In fact, ${ }^{6} \mathrm{Li}$ is rapidly consumed at stellar temperatures higher than $2 \times 10^{6} \mathrm{~K}$. The major source of ${ }^{6} \mathrm{Li}$ has been thought for decades to be the interaction of galactic cosmic rays with the interstellar medium [16]. The low energy capture reaction ${ }^{6} \mathrm{Li}(\mathrm{p}, \gamma){ }^{7} \mathrm{Be}$ plays an important role in the consumption of ${ }^{6} \mathrm{Li}$ and formation of ${ }^{7} \mathrm{Be}$.

The S-factor for this reaction is dominated by captures to the ground state and the 1 st excited state of ${ }^{7} \mathrm{Be}$. Both the ground state $\left(J_{b}=3 / 2^{-}\right)$ and the 1 st excited state $\left(J_{b}=1 / 2^{-}\right)$of ${ }^{7} \mathrm{Be}$ are described as a $j_{b}=p_{1 / 2}$ proton interacting with the ${ }^{6} \mathrm{Li}$ core, which has an intrinsic spin $I_{A}=1^{+}$. The parameters calculated according to Table I are used. The potential depths which reproduce the ground and excited states are given in Table II.

The continuum state potential depth for transitions to the ground state is set as $V_{c}=-37.70 \mathrm{MeV}$ following Ref. [17] and the corresponding one for the 1st excited is adjusted to fit the experimental S-factor for that capture (open circles in Fig. 2). In Ref. [17] the potential parameters and the spectroscopic factor for the ground state was obtained from a comparison between a finiterange distorted-wave Born approximation calculation and the experimental differential cross sections for the ${ }^{9} \mathrm{Be}\left({ }^{8} \mathrm{Li},{ }^{9} \mathrm{Be}\right){ }^{8} \mathrm{Li}$ elastic-transfer reaction at $27 \mathrm{MeV}$. The spectroscopic factors so obtained were compared with shellmodel calculations and other experimental values. The spectroscopic factor is 0.83 for the ground state following Ref. [17] and 0.84 for the 1st excited state, following Ref. [18].

In Ref. [18], the reaction is also compared with a calculation based on a four-cluster microscopic model. The energy dependence of the astrophysical S-factor for the ${ }^{6} \operatorname{Li}(\mathrm{p}, \gamma)^{7}$ Be reaction has been studied in Ref. [19], as well as in Ref. [20] where an analysis of the experimental data of Ref. [21] was done. It was found [20, 19] that the gamma-ray transition is dominated by the $E 1$ multipolarity and by incoming $s$ and $d$ waves.

Adopting the spectroscopic values listed above and including $s$ and $d$ incoming waves, we obtain the result shown in Fig. 2. Experimental data are from Ref. [22] (filled triangles), Ref. [23] (filled squares) and Ref. [18] (open circles). The agreement with the experimental data is very good and consistent with the previous studies [21, 20, 18, 17]. Based on these results, we obtain an ANC $\left(\sqrt{(S F) b^{2}}\right)$ of $2.01 \mathrm{fm}^{-1 / 2}$ for the ground state and 1.91 $\mathrm{fm}^{-1 / 2}$ for the 1 st excited state. 


\section{3. ${ }^{7} \operatorname{Li}(p, \gamma)^{8} \mathrm{Be}$}

The reaction ${ }^{7} \mathrm{Li}(\mathrm{p}, \gamma)^{8} \mathrm{Be}$ is part of the pp-chain in the Sun, leading to the formation of ${ }^{8} \mathrm{Be}[24]$. The unstable ${ }^{8} \mathrm{Be}$ decays into two $\alpha$-particles in $10^{-16}$ sec.

For this reaction, we consider only the capture to the ground state of ${ }^{8} \mathrm{Be}$ $\left(J_{b}=0^{+}\right)$, which is described as a $j_{b}=p_{3 / 2}$ proton coupled to the $I_{x}=3 / 2^{-}$ ${ }^{7} \mathrm{Li}$ core. The gamma-ray transition is dominated by the $E 1$ multipolarity and by incoming $s$ and $d$ waves. In order to reproduce the resonance at 0.386 $\mathrm{MeV}$ (in the c.m.), we choose a spectroscopic factor equal to 0.15. For the other resonance at $0.901 \mathrm{keV}$ (in the c.m.), we chose $S F=0.05$.

The result for both M1 resonances are shown in Figure 3, by dasheddotted curves. The potential depth for the continuum state, chosen as to reproduce the resonances, are $V_{c}=-46.35 \mathrm{MeV}$ and $V_{c}=-44.55 \mathrm{MeV}$, respectively. The non-resonant component (dashed-line) of the S-factor is obtained with $V_{c}=-56.69 \mathrm{MeV}$ and $S F=1.0$. The experimental data are from Ref. [25] (open circles). This reaction was also studied in Ref. [26]. They have obtained an spectroscopic factor of 0.4 for the first M1 resonance at $0.386 \mathrm{MeV}$ and $S F=1.0$ for the non-resonant capture. Their analysis is extended to angular distributions for the capture cross-section and analyzing power at $E_{p, l a b}=80 \mathrm{keV}$ which shows a strong E1-M1 interference, which helps to estimate the spectroscopic amplitudes.

If we only consider the fit to the non-resonant capture, our ANC $\left(\sqrt{(S F) b^{2}}\right)$ is $7.84 \mathrm{fm}^{-1 / 2}$. If we choose spectroscopic factors which reproduce the M1 resonances, the ANC-value evidently changes. This shows that the ANC extracted from radiative capture reactions with the use of a potential model are strongly dependent on the presence of resonances, specially those involving M1 transitions.

\section{4. ${ }^{7} B e(p, \gamma)^{8} B$}

The creation destruction of ${ }^{7} \mathrm{Be}$ in astrophysical environments is essential for understanding several stellar and cosmological processes and is not well understood. ${ }^{8} \mathrm{~B}$ also plays an essential role in understanding our Sun. High energy $\nu_{e}$ neutrinos produced by ${ }^{8} \mathrm{~B}$ decay in the Sun oscillate into other active species on their way to earth [27]. Precise predictions of the production rate of ${ }^{8} \mathrm{~B}$ solar neutrinos are important for testing solar models, and for limiting the allowed neutrino mixing parameters. The most uncertain reaction leading to ${ }^{8} \mathrm{~B}$ formation in the Sun is the ${ }^{7} \mathrm{Be}(\mathrm{p}, \gamma)^{8} \mathrm{~B}$ radiative capture reaction [28]. 
The $J_{b}=2^{+}$ground state of ${ }^{8} \mathrm{~B}$ is described as a $j_{b}=p_{3 / 2}$ proton coupled to the ${ }^{7}$ Be core, which has an intrinsic spin $I_{x}=3 / 2^{-}$. In this case, instead of the values in Table I, we take $a=0.52 \mathrm{fm}$ and $V_{s o}=-9.8 \mathrm{MeV}$. This is the same set of values adopted in Ref. [2]. The gamma-ray transition is dominated by the E1 multipolarity and by incoming $s$ and $d$ waves. The spectroscopic factor for non-resonant transitions is set to 1.0, which seems to reproduce best the $\mathrm{S}$-factor for this reaction at low energies. Our results are shown in Fig. 4. The experimental data are from Ref. [29] (open square), Ref. [30] (open circles), Ref. [31, 28, 32, 33] (solid triangle, open triangle, solid square, solid circle, solid diamond and open diamond).

In Ref. [28], the experimental data is reproduced with the cluster model calculation of Ref. [34] together with two incoherent Breit-Wigner resonances: a $1^{+} \mathrm{M} 1$ resonance at $0.63 \mathrm{MeV}$ fitted with $\Gamma_{p}=35.7 \pm 0.6 \mathrm{keV}$ and $\Gamma_{\gamma}=25.3 \pm 1.2 \mathrm{MeV}$, and a $3^{+}$resonance at $2.2 \mathrm{MeV}$ fitted with $\Gamma_{p}=350 \mathrm{keV}$ and $\Gamma_{\gamma}=150 \pm 30 \mathrm{MeV}$. Our calculated M1 resonance (dashed-dotted line) also reproduces well the data if we use $V_{c}=-38.14 \mathrm{MeV}$, and $S F=0.7$, with the other parameters according to Table I. For the non-resonant E1 transitions we use $V_{c}=-41.26 \mathrm{MeV}$ and $S F=1.0$. The S-factor at $E=0$, $S_{17}(0)$, is equal to $19.41 \mathrm{eV} . \mathrm{b}$, which is $10 \%$ smaller than that from the most recent experimental and theoretical analysis [28, 36].

A different experimental approach was used in Ref. [35], which extracted the ${ }^{8} \mathrm{~B}$ ANC from ${ }^{8} \mathrm{~B}$ breakup reactions at several energies and different targets. In that reference a slightly lower value of $S_{17}(0)=16.9 \pm 1.7 \mathrm{eV} . \mathrm{b}$ was inferred. That work also quotes an ANC of $0.67 \mathrm{fm}^{-1 / 2}\left(C^{2}=0.450(30)\right.$ $\left.\mathrm{fm}^{-1}\right)$. Our ANC, extracted from our fit to the radiative capture reaction, is $\sqrt{(S F) b^{2}}=0.72 \mathrm{fm}^{-1 / 2}$, not much different from Ref. [35].

\section{5. ${ }^{8} B(p, \gamma)^{9} C$}

Nucleosynthesis of light nuclei is hindered by the gaps at $A=5$ and $A=8$. The gap at $A=8$ may be bridged by reactions involving the unstable nuclei ${ }^{8} \mathrm{Li}\left(T_{1 / 2}=5840 \mathrm{~ms}\right)$ and ${ }^{8} \mathrm{~B}\left(T_{1 / 2}=5770 \mathrm{~ms}\right)$. The ${ }^{8} \mathrm{~B}(\mathrm{p}, \gamma){ }^{9} \mathrm{C}$ reaction breaks out to a hot part of the pp chain at temperatures such that this reaction becomes faster than the competing $\beta^{+}$decay. This reaction is especially relevant in low-metallicity stars with high masses where it can be faster than the triple- $\alpha$ process. It is also important under nova conditions. In both astrophysical scenarios this happens at temperatures several times larger than $10^{8} \mathrm{~K}$, corresponding to Gamow window energies around $E=$ $50-300 \mathrm{keV}$ [52, 38, 39]. 
The capture process for this reaction is dominated by E1 transitions from incoming s waves to bound p states [40] and the present work is restricted to an analysis of the capture to the ground state of ${ }^{9} \mathrm{C}\left(J_{b}=3 / 2^{-}\right)$, which is described as a $j_{b}=p_{3 / 2}$ proton coupled to the ${ }^{8} \mathrm{~B}$ core, which has an intrinsic spin $I_{x}=2^{+}$. The spectroscopic factor has been set to 1.0 as in Ref. [40], where several spectroscopic factor values are compared.

A renormalized folding potential for the continuum state is used in Ref. [40], while in our calculation $V_{c}$ is adjusted to $-22.55 \mathrm{MeV}$ to yield a similar result. This is done because there are no experimental data for this reaction. The results of both calculations are shown in Fig. 5. The open circle at $E=0$ is from Refs. [41, 42], which is an extrapolated value from a potential model using an ANC deduced from a breakup experiment. Ref. [40] also generates resonances by changing parameters of the folding potential. The ANC found in Ref. [43] is $1.15 \mathrm{fm}^{-1 / 2}\left(C^{2}=1.33 \pm 0.33 \mathrm{fm}^{-1}\right)$, whereas our $\operatorname{ANC}\left(\sqrt{(S F) b^{2}}\right)=1.31 \mathrm{fm}^{-1 / 2}$.

\section{6. ${ }^{9} \mathrm{Be}(p, \gamma){ }^{10} \mathrm{~B}$}

The reaction ${ }^{9} \mathrm{Be}(\mathrm{p}, \gamma){ }^{10} \mathrm{~B}$ plays an important role in primordial and stellar nucleosynthesis of light elements in the p-shell [1, 44]. Hydrogen burning in second generation stars occurs via the proton-proton (pp) chain and $\mathrm{CNO}$-cycle, with the ${ }^{9} \mathrm{Be}(\mathrm{p}, \gamma){ }^{10} \mathrm{~B}$ reaction serving as an intermediate link between these cycles

The $J_{b}=3^{+}$ground state of ${ }^{10} \mathrm{~B}$ is described as a $j_{b}=p_{3 / 2}$ proton coupled to the ${ }^{9} \mathrm{Be}$ core, which has an intrinsic spin $I_{A}=3 / 2^{-}$. The gammaray transition for the $\mathrm{DC}$ is dominated the $E 1$ multipolarity and by incoming $s$ waves. A spectroscopic factor $S F=1.0$ is used, which is the same value adopted in Ref. [45]. This value reproduces ${ }^{9} \mathrm{Be}(\mathrm{d}, \mathrm{n}){ }^{10} \mathrm{~B}$ and ${ }^{9} \mathrm{Be}\left({ }^{3} \mathrm{He}, \mathrm{d}\right){ }^{10} \mathrm{~B}$ reactions at incident energies of $10-20 \mathrm{MeV}$, and ${ }^{9} \mathrm{Be}(\alpha, \mathrm{t}){ }^{10} \mathrm{~B}$ at $65 \mathrm{MeV}$. It is also in accordance with the theoretical predictions of Refs. [46, 47].

The potential depth for the continuum state $V_{c}=-31.82 \mathrm{MeV}$ has been adjusted so that we can reproduce the direct capture measurements reported in Refs. [48]. It also reproduces the results of Ref. [49] where a reanalysis of the existing experimental data on ${ }^{9} \mathrm{Be}(\mathrm{p}, \gamma){ }^{10} \mathrm{~B}$ was done within the framework of the R-matrix method. The direct capture part of the S-factor was calculated using the experimentally measured ANC for ${ }^{10} \mathrm{~B} \rightarrow{ }^{9} \mathrm{Be}+\mathrm{p}$. The results are shown in Fig. 6. The experimental data are from Ref. [48] (filled squares). These data have also been fitted in Ref. [50] using R-matrix formulae that include channel contributions where appropriate. The curve passing 
through the experimental data points is the sum of our DC calculation and the resonance fits, given by the dashed lines.

In Ref. [51], the differential cross sections for the reactions ${ }^{9} \mathrm{Be}\left({ }^{10} \mathrm{~B},{ }^{10} \mathrm{~B}\right){ }^{9} \mathrm{Be}$ and ${ }^{9} \mathrm{Be}\left({ }^{10} \mathrm{~B},{ }^{9} \mathrm{Be}\right){ }^{10} \mathrm{~B}$ have been measured at an incident energy of $100 \mathrm{MeV}$. By normalizing the theoretical cross sections to the experimental data, the ANC for ${ }^{10} \mathrm{~B} \rightarrow{ }^{9} \mathrm{Be}+\mathrm{p}$ was extracted and found to be $2.22 \mathrm{fm}^{-1 / 2}\left(C^{2}=\right.$ $\left.4.91 \mathrm{fm}^{-1}\right)$, whereas the ANC $\left(\sqrt{(S F) b^{2}}\right)$ obtained from our fit to the previous analysis of Refs. [45, 50] is $3.43 \mathrm{fm}^{-1 / 2}$.

\section{7. ${ }^{11} C(p, \gamma){ }^{12} N$}

For first generation stars (those composed entirely of nuclei produced in the Big Bang) can only undergo nucleosynthesis via the pp chains or the triple-alpha process until heavier nuclei are produced to initiate the CNO cycle. For supermassive first generation stars, such processes generate too little energy and the triple-alpha reaction turns on too late to cause an explosion. Rather, such stars could simply collapse to black holes. However, hot pp chains provide a path for supermassive first generation stars to produce CNO nuclei at a lower temperature than required by the triple-alpha reaction [52]. These CNO nuclei then serve as seeds for further energy generation, stablizing the star against collapse long enough to permit an explosion to occur. Both the ${ }^{8} \mathrm{~B}(\mathrm{p}, \gamma){ }^{9} \mathrm{C}$ and ${ }^{11} \mathrm{C}(\mathrm{p}, \gamma){ }^{12} \mathrm{~N}$ reactions are important in the hot pp chains.

For the ${ }^{11} \mathrm{C}(\mathrm{p}, \gamma){ }^{12} \mathrm{~N}$ reaction, non-resonant capture into the ground state of ${ }^{12} \mathrm{~N}$ and resonant capture into the first and second excited states is thought to dominate the reaction rate at stellar energies [53]. There are no experimental data for this reaction, except for indirect determination of the astrophysical S-factors, e.g. by using the ANC for ${ }^{12} \mathrm{~N} \rightarrow{ }^{11} \mathrm{C}+\mathrm{p}$ from the ${ }^{14} \mathrm{~N}\left({ }^{11} \mathrm{C},{ }^{12} \mathrm{~N}\right){ }^{13} \mathrm{C}$ peripheral transfer reaction. Another indirect measurement for the astrophysical rate of the ${ }^{11} \mathrm{C}+\mathrm{p}$ reaction was obtained from from the Coulomb break-up of a ${ }^{12} \mathrm{~N}$ radioactive beam in Ref. [54].

The ground state of ${ }^{12} \mathrm{~N}\left(J_{b}=1^{+}\right)$is described as a $j_{b}=p_{1 / 2}$ proton coupled to the ${ }^{11} \mathrm{C}$ core, which has an intrinsic spin $I_{x}=3 / 2^{-}$. The direct capture gamma-ray transition is dominated by the $E 1$ multipolarity and by incoming $s$ waves. The spectroscopic factor has been set to 0.4 , the same value used in Ref. [54] (0.4 \pm 0.25$)$.

The result for our DC calculation is shown in Fig. 77. Similar results have also been reported in Refs. [53, 54, 55], and in Ref. [56] which also used the ANC method to extract the low-nergy S-factor via measurement of ${ }^{11} \mathrm{C}(\mathrm{d}$, 
$\mathrm{n})^{12} \mathrm{~N}$ reaction. The ANC found in Ref. [53] is $1.32 \mathrm{fm}^{-1 / 2}\left(C^{2}=1.73 \pm 0.25\right.$ $\left.\mathrm{fm}^{-1}\right)$ and in Ref. [56] is $1.69 \mathrm{fm}^{-1}\left(C^{2}=2.86 \pm 0.91 \mathrm{fm}^{-1}\right)$. Our ANC value is $\sqrt{(S F) b^{2}}=0.94 \mathrm{fm}^{-1 / 2}$.

\section{8. ${ }^{12} C(p, \gamma){ }^{13} N$}

The abundance ratio ${ }^{12} \mathrm{C} /{ }^{13} \mathrm{C}$ is an important measure of stellar evolution and nucleosynthesis. Changes in ratios of ${ }^{12} \mathrm{C} /{ }^{13} \mathrm{C}$ in stars happen as they evolve from the main sequence to the first ascent giant branch. Later, the convection zone grows and penetrates to greater depths where it begins to dredge up material that has been hot enough for the CNO cycle to convert to $\mathrm{N}$. This is when the primordial ${ }^{12} \mathrm{C}$ is converted into ${ }^{13} \mathrm{C}$ and ${ }^{14} \mathrm{~N}$ by the reactions ${ }^{12} \mathrm{C}(\mathrm{p}, \gamma){ }^{13} \mathrm{~N}\left(\beta^{+}\right){ }^{13} \mathrm{C}$ and ${ }^{13} \mathrm{C}(\mathrm{p}, \gamma){ }^{14} \mathrm{~N}$, hence reducing the ${ }^{12} \mathrm{C} /{ }^{13} \mathrm{C}$ ratio. During the late asymptotic giant branch AGB phase, the stars suffer thermal instabilities in the helium shell where partial helium burning occurs causing the ${ }^{12} \mathrm{C} /{ }^{13} \mathrm{C}$ ratio to increase [1].

The ${ }^{12} \mathrm{C}(\mathrm{p}, \gamma){ }^{13} \mathrm{~N}$ direct capture to the ground state proceeds primarily through E1 $\left(s_{1 / 2} \rightarrow p_{1 / 2}\right)$ and E1 $\left(d_{3 / 2} \rightarrow p_{1 / 2}\right)$ single-particle transitions [57]. The ground state of ${ }^{13} \mathrm{~N}\left(J_{b}=1 / 2^{-}\right)$is described as a $j_{b}=p_{1 / 2}$ proton coupled to the ${ }^{12} \mathrm{C}$ core, which has an intrinsic spin $I_{x}=0^{+}$.

Experimental cross sections for the ${ }^{12} \mathrm{C}(\mathrm{p}, \gamma)$ capture to the ground state of ${ }^{13} \mathrm{~N}$ were published in Ref. [58]. Choosing the spectroscopic factor as $S F=1$, leads to the dashed line shown in Fig. 8, if we use the same potential depth as for the bound state. The E1 resonance at $0.422 \mathrm{MeV}$ [57] is generated when we choose $V_{c}=-53.50 \mathrm{MeV}$ and a spectroscopic factor equal to 0.35. The result for the resonance is shown as a dotted-line in Fig. 8. The total S-factor is shown by a solid line.

The resonance at $0.422 \mathrm{MeV}$ (c.m.) has also been studied experimentally and theoretically in Refs. [61, 60, 59]. The ANC obtained in Refs. [62, 63, 60] is $1.43 \pm 0.09 \mathrm{fm}^{-1 / 2}$, whereas our $\mathrm{ANC}\left(\sqrt{(S F) b^{2}}\right)$, corresponding to the non-resonant capture, is $2.05 \mathrm{fm}^{-1 / 2}$.

\section{9. ${ }^{13} C(p, \gamma){ }^{14} N$}

This reaction is another important reaction in the $\mathrm{CNO}$ cycle. It precedes the slowest reaction in the $\mathrm{CNO}$ cycle, the ${ }^{14} \mathrm{~N}(\mathrm{p}, \gamma){ }^{15} \mathrm{O}$ radiative capture reaction, which defines the rate of energy production in the cycle. The ${ }^{13} \mathrm{C}(\mathrm{p}$, $\gamma)^{14} \mathrm{~N}$ radiative capture rate is also important for nucleosynthesis via the slow proton capture process because it depletes the seed nuclei required for the 
neutron generator reaction ${ }^{13} \mathrm{C}(\alpha, \mathrm{n}){ }^{16} \mathrm{O}$ in AGB stars with solar metalicity [64, 65].

Extensive experimental data on this reaction was published in Ref. [66]. One concludes that this capture is dominated by transitions to the ground state. The direct capture to the ground state proceeds primarily through E1 $\left(s_{1 / 2} \rightarrow p_{1 / 2}\right)$ and E1 $\left(d_{3 / 2} \rightarrow p_{1 / 2}\right)$ single-particle transitions [57]. The ground state of ${ }^{14} \mathrm{~N}\left(J_{b}=1^{+}\right)$is described as a $j_{b}=p_{1 / 2}$ proton coupled to the ${ }^{13} \mathrm{C}$ core, which has an intrinsic spin $I_{x}=1 / 2^{-}$.

We could not reproduce the E1 resonance at $E_{c m}=0.518 \mathrm{MeV}$ using the potential parameters of Ref. [57]. In fact, we notice that figure 5 of Ref. [57] is inconsistent with its caption (center of mass and laboratory systems are swapped). In figure 9, the dotted line is our calculation for the resonance, which is obtained with the parameters from Table I and with generated when $V_{c}=-50.60 \mathrm{MeV}$ and spectroscopic factor 0.15 . For non-resonant capture, the potential depth for the continuum state has been chosen as $V_{c}=-44.10$ $\mathrm{MeV}$ to reproduce the same result as in Ref. [57]. The spectroscopic factor has been set to 0.33 following Ref. [66]. The non-resonant calculation is shown by a dashed line in Fig. 9. The total S-factor is shown as a solid line.

In Ref. [67], the ${ }^{13} \mathrm{C}(\mathrm{p}, \gamma){ }^{14} \mathrm{~N}$ radiative capture reaction is analyzed within the R-matrix approach. The experimental ANCs induced from the ${ }^{13} \mathrm{C}\left({ }^{14} \mathrm{~N},{ }^{13} \mathrm{C}\right){ }^{14} \mathrm{~N}$ and ${ }^{13} \mathrm{C}\left({ }^{3} \mathrm{He}, \mathrm{d}\right){ }^{14} \mathrm{~N}$ reactions are used in the analysis. The obtained ANC is $4.3 \mathrm{fm}^{-1 / 2}\left(C^{2}=18.2 \mathrm{fm}^{-1}\right)$, whereas our ANC value is $\sqrt{(S F) b^{2}}=3.05 \mathrm{fm}^{-1 / 2}$.

3.10. ${ }^{13} N(p, \gamma)^{14} \mathrm{O}$

For temperatures up to $10^{9} \mathrm{~K}\left(T_{9}=1\right)$, this reaction is vital for understanding hydrogen burning in the hot $\mathrm{CNO}$ cycle and the conditions under which break-out into the rp-process might occur.

The ground state of ${ }^{14} \mathrm{O}\left(J_{b}=0^{+}\right)$is described as a $j_{b}=p_{1 / 2}$ proton coupled to the ${ }^{13} \mathrm{~N}$ core, which has an intrinsic spin $I_{x}=1 / 2^{-}$. The gamma-ray transition for the DC to the ground state is dominated the $E 1$ multipolarity and by incoming $s$ waves. For the non-resonant capture (lower curves in figure [10), if we choose the potential depth for the continuum state to be the same as that for the bound state $\left(V_{c}=V_{b}\right)$, we obtain S-factors which are up to 3 times larger than the one in Ref. [68] where a theoretical description of this reaction has been reported. We find that $V_{c}=-25.20 \mathrm{MeV}$ reproduces rather well the non-resonant capture calculation of Ref. 68. The spectroscopic factor has been set to 1.88 following Ref. [69], where a DWBA analysis 
of the ${ }^{13} \mathrm{~N}(\mathrm{~d}, \mathrm{n}){ }^{14} \mathrm{O}$ reaction at $E_{c m}=8.9 \mathrm{MeV}$ was used to obtain the ANC for the ground state of ${ }^{14} \mathrm{O} \longrightarrow{ }^{13} \mathrm{~N}+$ p. Our non-resonant DC calculation is shown as a dotted-dashed line in Fig. 10.

We reproduce the E1 resonance at $0.528 \mathrm{MeV}$ (s wave capture, according to Ref. [71]) as shown by the solid line in figure 10 if we choose $V_{c}=$ $-52.14 \mathrm{MeV}$ and $S F=1.0$. Very similar results were obtained in Refs. [73, 72, 70, 71, 69, 61]. The ANCs reported in there publications are 5.51 $\mathrm{fm}^{-1 / 2}\left(C^{2}=30.4 \pm 7.1 \mathrm{fm}^{-1}\right)[68], 5.42 \pm 0.48 \mathrm{fm}^{-1 / 2}[69]$ and $5.39 \mathrm{fm}^{-1 / 2}$ $\left(C^{2}=29.0 \pm 4.3 \mathrm{fm}^{-1}\right)[71]$. In Ref. [71] the radiative capture cross section was estimated using an R-matrix approach with the measured ANC from the ${ }^{14} \mathrm{~N}\left({ }^{13} \mathrm{~N},{ }^{14} \mathrm{O}\right){ }^{13} \mathrm{C}$ peripheral transfer reaction at $11.8 \mathrm{MeV} /$ nucleon incident energy. We obtain the ANC value $\sqrt{(S F) b^{2}}=5.44 \mathrm{fm}^{-1 / 2}$, which is in accordance with these results.

\subsection{1. ${ }^{14} N(p, \gamma)^{15} \mathrm{O}$}

At astrophysical energies, this reaction is the slowest process in the hydrogen burning $\mathrm{CNO}$ cycle [1]. It plays a key role in the energy production of more massive main sequence stars and the detailed understanding of the neutrino spectrum of our sun [74, 75] as well as the age determination of globular cluster stars [76].

The main contribution to the S-factor for this reaction is due to the transition to the $6.793 \mathrm{MeV}$ excited state of ${ }^{15} \mathrm{O}\left(J_{b}=3 / 2^{+}\right)$[78, 77], which is described as a $j_{b}=s_{1 / 2}$ proton coupled to the ${ }^{14} \mathrm{~N}$ core $\left(I_{x}=1^{+}\right)$. The gamma-ray transition is dominated the $E 1$ multipolarity and by incoming $p$ waves. In this case, instead of the parameters of Table 1, we use $r_{0}=1.3 \mathrm{fm}$, $r_{c}=1.2, a=0.6 \mathrm{fm}$ and $V_{s 0}=-2.0 \mathrm{MeV}$, which were also adopted in Ref. [79]. The spectroscopic factor is $S F=1.5$. The result of our calculation is shown by the dashed line in Fig. 11 .

Ref. [80] discusses experiments on stripping reactions and theoretical shell-model calculations used to describe the ${ }^{14} \mathrm{~N}(\mathrm{p}, \gamma){ }^{15} \mathrm{O}$ radiative capture. These studies indicate that the resonance at $0.259 \mathrm{MeV}$ decays to the 6.793 $\mathrm{MeV}$ excited state of ${ }^{15} \mathrm{O}$ via a $\mathrm{M} 1$ radiation. To describe this resonance, we use the same spectroscopic factor, $S F=0.51$, as obtained experimentally in Ref. 81] where spectroscopic factors and ANCs have been determined for bound states in ${ }^{15} \mathrm{O}$ using the ${ }^{14} \mathrm{~N}\left({ }^{3} \mathrm{He}, \mathrm{d}\right){ }^{15} \mathrm{O}$ reaction. Several other spectroscopic values from the literature were also discussed in Ref. [81]. We found an optimal value for the potential depth to be $V_{c}=-49.18 \mathrm{MeV}$. Adopting this value, our calculations yield the dotted line shown in the figure. 
The total S-factor is shown as a solid line. Experimental data are from Ref. [78] (filled squares), Ref. [82] (filled triangles), Ref. [83] (open circles). The R-matrix method was used to study this reaction in Ref. [78] and Ref. [84]. The ANC found in Ref. [85], deduced from the ${ }^{13} \mathrm{C}\left({ }^{14} \mathrm{~N},{ }^{13} \mathrm{C}\right){ }^{14} \mathrm{~N}$ and ${ }^{13} \mathrm{C}\left({ }^{3} \mathrm{He}, \mathrm{d}\right){ }^{14} \mathrm{~N}$ reactions, is $4.90 \mathrm{fm}^{-1 / 2}\left(C^{2}=24 \pm 5 \mathrm{fm}^{-1}\right)$. Ref. [81] adopts the value $4.6 \mathrm{fm}^{-1 / 2}\left(C^{2}=21 \pm 5 \mathrm{fm}^{-1}\right)$. Our ANC obtained from the DC fitting is $\sqrt{(S F) b^{2}}=5.19 \mathrm{fm}^{-1 / 2}$.

\subsection{2. ${ }^{15} \mathrm{~N}(p, \gamma){ }^{16} \mathrm{O}$}

In second-generation stars with masses larger than the mass of the Sun, hydrogen burning proceeds predominantly through the CNO cycle [1]. The main sequence of reaction leads to an energy release of $25 \mathrm{MeV}$ per cycle. There is a loss of $\mathrm{CN}$ catalyst from this cycle through the reaction ${ }^{15} \mathrm{~N}(\mathrm{p}$, $\gamma)^{16} \mathrm{O}$. This is replenished by a sequence of reactions involving oxygen and fluorine, leading to the formation of ${ }^{14} \mathrm{~N}$ and ${ }^{15} \mathrm{~N}$. The reaction rate of ${ }^{15} \mathrm{~N}(\mathrm{p}$, $\gamma{ }^{16} \mathrm{O}$ determines the overall abundance of the oxygen isotopes synthesized in the CNO tri-cycle [1] and therefore plays an important role in stellar nucleosynthesis.

This reaction is dominated by the capture into the ground state of ${ }^{16} \mathrm{O}$ $\left(J_{b}=0^{+}\right)$[86], which is described as a $j_{b}=p_{1 / 2}$ proton coupled to the ${ }^{15} \mathrm{~N}$ core $\left(I_{x}=1 / 2^{-}\right)$. The reaction is dominated by resonant capture to the ground state through the first two interfering $J^{\pi}=1^{-}$s-wave resonances at $E_{c m}=312$ and $964 \mathrm{keV}$

We will restrict ourselves to the non-resonant capture to the ground state, as a good reproduction of the resonances is not possible with the simple potential model. The non-resonant capture process proceeds predominantly through an E1 $(s \rightarrow p)$ transition [86]. A spectroscopic factor $S F=1.8$ is used for the ground state of ${ }^{16} \mathrm{O}$, following Ref. [86] which studied the excitation functions of this reaction at $E_{p}=150-2500 \mathrm{keV}$. This value is also in accordance with Ref. [87].

Our calculation is shown in Fig. 12, Experimental data are from Ref. [86] (filled triangles), Ref. [91] (filled squares). Ref. [88] extracted ANCs from the differential cross sections for the ${ }^{15} \mathrm{~N}\left({ }^{3} \mathrm{He}, \mathrm{d}\right){ }^{16} \mathrm{O}$ reaction. Using these ANCs and proton and $\alpha$-resonance widths determined from an R-matrix fit to the data from the ${ }^{15} \mathrm{~N}(\mathrm{p}, \alpha){ }^{12} \mathrm{C}$ reaction, the astrophysical factor for ${ }^{15} \mathrm{~N}(\mathrm{p}, \gamma){ }^{16} \mathrm{O}$ was obtained. The results from Ref. [86] and Ref. [88] are also shown in Fig. [12, In Ref. [86], the resonances are described by using a fit with single level Breit-Wigner shapes. The ANC found in Ref. [88] is 13.86 
$\mathrm{fm}^{-1 / 2}\left(C^{2}=192.0 \pm 26.0 \mathrm{fm}^{-1}\right)$. Our ANC is very close to this value, i.e., $\sqrt{(S F) b^{2}}=13.63 \mathrm{fm}^{-1 / 2}$.

\subsection{3. ${ }^{16} O(p, \gamma){ }^{17} \mathrm{~F}$}

Many stars, including the Sun, will eventually pass through an evolutionary phase that is referred to as the asymptotic giant branch (AGB) [89]. This phase involves a hydrogen and a helium shell that burn alternately surrounding an inactive stellar core. The ${ }^{16} \mathrm{O}(\mathrm{p}, \gamma){ }^{17} \mathrm{~F}$ reaction rate influences sensitively the ${ }^{17} \mathrm{O} /{ }^{16} \mathrm{O}$ isotopic ratio predicted by models of massive $\left(\geq 4 M_{\odot}\right)$ AGB stars, where proton captures occur at the base of the convective envelope (hot bottom burning). A fine-tuning of the ${ }^{16} \mathrm{O}(\mathrm{p}, \gamma){ }^{17} \mathrm{~F}$ reaction rate may account for the measured anomalous ${ }^{17} \mathrm{O} /{ }^{16} \mathrm{O}$ abundance ratio in small grains with are formed by the condensation of the material ejected from the surface of AGB stars via strong stellar winds [90].

We calculate the capture to the ground state and to the 1st excited state of ${ }^{17} \mathrm{~F}$. The $J_{b}=5 / 2^{+}$ground state $\left(J_{b}=1 / 2^{+}\right.$excited state ${ }^{17} \mathrm{~F}$ is described as a $j_{b}=d_{5 / 2}$ neutron $\left(j_{b}=s_{1 / 2}\right.$ neutron) coupled to the ${ }^{16} \mathrm{O}$ core, which has an intrinsic spin $I_{x}=0^{+}$. In this case, the values $a=0.6 \mathrm{fm}$ and $R_{C}=R=3.27 \mathrm{fm}$ are adopted, which are the same values used in Ref. [92]. The gamma-ray transitions are dominated by the $E 1$ multipolarity and by incoming $p$ waves for both states. The M1 and E2 contributions amount to less than $0.1 \%$ of the dominant E1 contribution, as shown in Ref. [93] where a potential model was also used.

We use spectroscopic factors equal to 0.9 and 1.0 for the ground state and the excited state, respectively, following Ref. [93]. Our results are shown in Fig. 13. The experimental data are from Ref. [94] (filled squares), Ref. [95] (filled triangles), Ref. [93] (open circles), and Ref. [96] (open triangles).

Ref. [98] reports a study of the ${ }^{16} \mathrm{O}\left({ }^{3} \mathrm{He}, \mathrm{d}\right){ }^{17} \mathrm{~F}$ reaction to determine ANCs for transitions to the ground and first excited states of ${ }^{17} \mathrm{~F}$. The ANCs found in Ref. [98] are $1.04 \mathrm{fm}^{-1 / 2}\left(C^{2}=1.08 \pm 0.1 \mathrm{fm}^{-1}\right)$ for the ground state, and $80.6 \mathrm{fm}^{-1 / 2}\left(C^{2}=6490 \pm 680 \mathrm{fm}^{-1}\right)$ for the first excited state of ${ }^{17} \mathrm{~F}$, respectively. Our ANC values are $\sqrt{(S F) b^{2}}=0.91 \mathrm{fm}^{-1 / 2}$ for the ground state and $77.21 \mathrm{fm}^{-1 / 2}$ for the 1 st excited state.

\subsection{4. ${ }^{20} \mathrm{Ne}(p, \gamma)^{21} \mathrm{Na}$}

Along with the p-p chain and the CNO tri-cycle, the Ne-Na cycle [99] is also of importance in hydrogen burning in second-generation stars with

masses larger than the mass of the Sun. The ${ }^{20} \mathrm{Ne}(\mathrm{p}, \gamma)^{21} \mathrm{Na}$ reaction is the 
first reaction of the cycle. The nuclei ${ }^{21} \mathrm{Na},{ }^{21} \mathrm{Ne},{ }^{22} \mathrm{Na},{ }^{22} \mathrm{Ne}$, and ${ }^{23} \mathrm{Na}$ are gradually created during $\mathrm{Ne}-\mathrm{Na}$ burning. ${ }^{21} \mathrm{Ne}$ is of additional interest for subsequent He burning in stars. Due to the positive Q-value of $2.56 \mathrm{MeV}$ for the ${ }^{21} \mathrm{Ne}(\alpha, \mathrm{n}){ }^{24} \mathrm{Mg}$ reaction, ${ }^{21} \mathrm{Ne}$ can act as a source of neutrons. Subsequent capture of these neutrons contributes to the synthesis of the heavier elements [99].

As observed in Ref. [100], the direct capture to the $2.425 \mathrm{MeV}\left(J^{\pi}=\right.$ $\left.1 / 2^{+}\right)$and $0.332 \mathrm{MeV}\left(J^{\pi}=5 / 2^{+}\right)$excited state dominate the total S-factor for this reaction. The $J_{b}=1 / 2^{+}$excited state $\left(J_{b}=5 / 2^{+}\right.$excited state) of ${ }^{21} \mathrm{Na}$ is described as a $j_{b}=s_{1 / 2}$ proton $\left(j_{b}=d_{5 / 2}\right.$ proton) interacting with the ${ }^{20} \mathrm{Ne}$ core, which has an intrinsic spin $I_{x}=0^{+}$. The gamma-ray transition is dominated by the $E 1$ multipolarity and by incoming $p$ waves.

The spectroscopic factor obtained in Ref. [100] is 0.9. More recently, Ref. [101] determined the ANC for ${ }^{21} \mathrm{Na} \rightarrow{ }^{20} \mathrm{Ne}+\mathrm{p}$ from the analysis of ${ }^{20} \mathrm{Ne}\left({ }^{3} \mathrm{He}\right.$, d $)^{21} \mathrm{Na}$ proton transfer reaction at an incident energy of $25.83 \mathrm{MeV}$, and obtained the spectroscopic factor of 0.6. We used the spectroscopic factor $S F=0.7$ for the $2.425 \mathrm{MeV}$ excited state and $S F=0.8$ for the $0.332 \mathrm{MeV}$ excited state, which are values between those of Refs. [100] and [101]. Our results are shown in Fig. 14. Experimental data are from Ref. [100].

For the $2.425 \mathrm{MeV}$ excited stated, the ANC found in Ref. [101] is $8.29 \times$ $10^{16} \mathrm{fm}^{-1 / 2}\left(C^{2}=6.8694 \times 10^{33} \mathrm{fm}^{-1}\right)$, whereas our computed ANC value is

$\sqrt{(S F) b^{2}}=3.36 \mathrm{fm}^{-1 / 2}$. The reason for this large discrepancy is not clear. It might be, as seen from Fig. 14, due to the steep slope of the S-factor at low energies. This points to a subthreshold resonance and a possible large sensitivity of the ANC for this state. On the other hand, for the $0.332 \mathrm{MeV}$ excited state, the ANC found in Ref. [101] is $1.55 \mathrm{fm}^{-1 / 2}\left(C^{2}=2.41 \mathrm{fm}^{-1}\right)$, whereas our computed ANC value is $\sqrt{(S F) b^{2}}=2.17 \mathrm{fm}^{-1 / 2}$.

\section{Neutron capture}

Table 3 summarizes the potential parameters used in the cases where the single-particle model works reasonably well in calculating radiative neutron capture reactions. A discussion is presented case by case in the following subsections. Unless otherwise stated, we use the parameters described in Table 1 for the single-particle potential. The parameters for the continuum potential, $V_{c}$, are the same as those for the bound state potential, except for the few cases explicitly discussed in the text. 


\section{1. ${ }^{2} H(n, \gamma)^{3} H$}

The ${ }^{2} \mathrm{H}(\mathrm{p}, \gamma)^{3} \mathrm{He}$ reaction at low energies, followed by $\mathrm{d}\left({ }^{3} \mathrm{He}, \mathrm{p}\right)^{4} \mathrm{He}$, leads to the formation of ${ }^{4} \mathrm{He}$ during the primordial nucleosynthesis era [102, 103, 104]. It also plays a key role during the proto-stars era, in which the energy generated by deuterium burning slowed down the contraction due to the gravitational force [105, 106]. On the other hand, the ${ }^{2} \mathrm{H}(\mathrm{n}, \gamma)^{3} \mathrm{H}$ reaction is thought to contribute to inhomogeneous big-bang models [107, 109, 108]. These models assume the existence of neutron-rich and neutron-poor regions resulting from a first-order phase transition from quarks to hadrons as the universe cooled down [107]. In the neutron-rich region, reactions such as ${ }^{2} \mathrm{H}(\mathrm{n}, \gamma){ }^{3} \mathrm{H}(\mathrm{d}, \mathrm{n}){ }^{4} \mathrm{He}\left({ }^{3} \mathrm{H}, \gamma\right){ }^{7} \operatorname{Li}(\mathrm{n}, \gamma){ }^{8} \operatorname{Li}(\alpha, \mathrm{n}){ }^{11} \mathrm{~B}(\mathrm{n}, \gamma){ }^{12} \mathrm{~B}$, produce an appreciable amount of intermediate-heavy nuclei.

We consider only the E1 capture to the ground state of ${ }^{3} \mathrm{H}(p \rightarrow s)$. The $J_{b}=1 / 2^{+}$ground state ${ }^{3} \mathrm{He}$ is described as a $j_{b}=s_{1 / 2}$ neutron coupled to the ${ }^{2} \mathrm{H}$ core, which has an intrinsic spin $I_{x}=1^{+}$.

The calculation for this reaction requires a three-body treatment which is beyond the scope of this work. Obviously, the potential model adopted here is oversimplified for this case. We choose an spectroscopic factor equal to $S F=1.0$. Our results are shown in Fig. 15, where the dashed and dash-dotted curves are the evaluated reaction rates presented in Ref. [110] based on a phenomenological parametrization of the cross section based on evaluated nuclear data tables. The experimental data are from Ref. [110]. In Ref. [110] the neutron-deuteron capture was obtained using time reversal from the two-body photodisintegration amplitude and wavefunctions obtained with the AV18 potential [111] alone or combined with the Urbana IX three-nucleon force [112]. Their results are shown by the open (solid) triangles with (without) the three-body nn interaction. The ANC calculated with our potential model is $\sqrt{(S F) b^{2}}=1.90 \mathrm{fm}^{-1 / 2}$.

\section{2. ${ }^{7} \mathrm{Li}(n, \gamma)^{8} \mathrm{Li}$}

The ${ }^{7} \mathrm{Li}(\mathrm{n}, \gamma)^{8} \mathrm{Li}$ cross section is often used to extrapolate the capture cross section for the reaction ${ }^{7} \mathrm{Be}(\mathrm{p}, \gamma)^{8} \mathrm{~B}$ down to the solar energies at $E_{c m} \sim 20$ $\mathrm{keV}$, which is relevant for the production of high energy neutrinos in the Sun [113]. The ${ }^{7} \mathrm{Li}(\mathrm{n}, \gamma)^{8} \mathrm{Li}$ reaction is also relevant for the rapid process during primordial nucleosynthesis of nuclei with $A>12$ in the inhomogeneous bigbang models [108, 114]. In these models, the main reaction chain leading to the synthesis of heavy elements is $108{ }^{1} \mathrm{H}(\mathrm{n}, \gamma)^{2} \mathrm{H}(\mathrm{n}, \gamma)^{3} \mathrm{H}(\mathrm{d}, \mathrm{n})^{4} \mathrm{He}(\mathrm{t}, \gamma)^{7} \mathrm{Li}(\mathrm{n}$, $\gamma)^{8} \mathrm{Li}$, and then ${ }^{8} \mathrm{Li}(\alpha, \mathrm{n})^{11} \mathrm{~B}(\mathrm{n}, \gamma)^{12} \mathrm{~B}\left(\beta^{-}\right){ }^{12} \mathrm{C}(\mathrm{n}, \gamma){ }^{13} \mathrm{C}$, etc., for heavier nuclei. 
The reaction ${ }^{7} \mathrm{Li}(\mathrm{n}, \gamma){ }^{8} \mathrm{Li}$ is thus a crucial input to bridge the gap of mass $A=8$, leading to the production of heavy elements.

We consider the capture to the ground state and to the first excited state of ${ }^{8} \mathrm{Li}$. A similar calculation has been done in Ref. [115], where the partial cross sections from neutron capture to the ground and first excited states in ${ }^{8} \mathrm{Li}$ at stellar energies were reported. The gamma-ray transitions are dominated by the $E 1$ multipolarity and by incoming $s$ waves and $d$ waves. The $J_{b}=2^{+}$ground state $\left(J_{b}=1^{+}\right.$first excited state) of ${ }^{8} \mathrm{Li}$ is described as a $j_{b}=p_{3 / 2}$ neutron interacting with the ${ }^{7} \mathrm{Li}$ core, which has an intrinsic spin $I_{x}=3 / 2^{-}$.

In this particular case, the values $R_{0}=R_{C}=R_{S 0}=2.391 \mathrm{fm}$ are used. For the continuum state, the potential depth has been adjusted to reproduce the experimental scattering lengths $a_{+}=-3.63 \pm 0.05 \mathrm{fm}$ and $a_{-}=+0.87 \pm$ $0.05 \mathrm{fm}$ for the two components of the channel spin $s$ at thermal energies. The resulting potential depth parameters are $V_{c}=-56.15 \mathrm{MeV}$ and $V_{c}=-46.50$ $\mathrm{MeV}$, for the $s=2$ and $s=1$ spin components, respectively. Following Ref. [115], we use the spectroscopic factors $S F(g . s)=$.0.87 and $S F(1 s t)=0.48$, for the ground and first excited states, respectively. The capture to the first excited state contributes to less than $5 \%$ of the total cross section. The M1 resonance at $E_{R}=0.26 \mathrm{MeV}$ for capture to the ground state is reproduced with $V_{c}=-34.93 \mathrm{MeV}$ and a spectroscopic factor $S F=1.0$.

The results of this calculation are shown in Fig. 16. The dashed and dotted lines are for the capture to the ground state and first excited state, respectively. Adding them together with the dashed-dotted line for the M1 resonance, one gets the total S-factor shown by the solid line. The experimental data are from refs. [116] (filled circles), [115] (filled triangles), [117] (filled squares), [118] (open circles) and [119] (open triangles). Our calculated ANC is $\sqrt{(S F) b^{2}}=0.71 \mathrm{fm}^{-1 / 2}$ for the ground state and $0.33 \mathrm{fm}^{-1 / 2}$ for the 1 st excited state of ${ }^{8} \mathrm{Li}$.

\section{3. ${ }^{8} \operatorname{Li}(n, \gamma){ }^{9} \mathrm{Li}$}

Rapid capture processes (r-processes) might occur in the post-collapse of a type II supernova, leading to the formation of heavy elements. Starting with a He-rich environment the mass- 8 gap is bridged by either $\alpha+\alpha+$ $\alpha \rightarrow{ }^{12} \mathrm{C}$ or $\alpha+\alpha+n \rightarrow{ }^{9} \mathrm{Be}$ reactions. During this process, a neutronrich freeze out occurs which triggers the r-process [121]. At this stage, it would also be possible to bridge the $A=8$ gap through the reaction chain 
${ }^{4} \mathrm{He}(2 \mathrm{n}, \gamma){ }^{6} \mathrm{He}(2 \mathrm{n}, \gamma){ }^{8} \mathrm{He}\left(\beta^{-}\right)^{8} \operatorname{Li}(\mathrm{n}, \gamma){ }^{9} \operatorname{Li}\left(\beta^{-}\right){ }^{9} \mathrm{Be}[122,123]$. This chain provides an alternative path to proceed along the neutron-rich side of the line of stability towards heavier isotopes. One needs to know to what extent this chain competes with the ${ }^{8} \mathrm{Li}\left(\beta^{-}\right)^{8} \mathrm{Be}(2 \alpha)$ process. An important clue to the answer depends on an accurate knowledge of the ${ }^{8} \mathrm{Li}(\mathrm{n}, \gamma){ }^{9} \mathrm{Li}$ reaction rate.

We consider the E1 $s$ - and $d$-wave captures to both the ground and the 1st excited state of ${ }^{9} \mathrm{Li}$. The $J_{b}=3 / 2^{-}$ground state and $J_{b}=1 / 2^{-} 1$ st excited state in ${ }^{9} \mathrm{Li}$ are described as a $j_{b}=p_{3 / 2}$ neutron coupled to the ${ }^{8} \mathrm{Li}$ core, which has an intrinsic spin $I_{x}=2^{+}$. Here we use $a=0.52 \mathrm{fm}, R=2.499 \mathrm{fm}$ and $V_{s o}=-9.9 \mathrm{MeV}$, which are adopted from Ref. [124]. The spectroscopic factors used in Ref. [125] are 1.65 and 0.55 for the ground and 1st excited state, respectively. However, for the ground state, most of experiments and calculations give $S F \approx 0.8$ (see the summary in Ref. [126]). Thus we use $S F=0.8$ instead of 1.65 for the ground state. The result is shown in Fig. 17. The experimental data are from Ref. [127] using the Coulomb dissociation of ${ }^{9} \mathrm{Li}$ on $\mathrm{Pb}$ targets at $28.5 \mathrm{MeV} / \mathrm{A}$ beam energy. From the result one can see the capture to the excited state is much weaker than that to the ground state. Our ANC $\left(\sqrt{(S F) b^{2}}\right)$ is $1.12 \mathrm{fm}^{-1 / 2}$ for the ground state of ${ }^{9} \mathrm{Li}$ and $0.40 \mathrm{fm}^{-1 / 2}$ for the 1 st excited state of ${ }^{9} \mathrm{Li}$.

\section{4. ${ }^{11} B(n, \gamma){ }^{12} B$}

Nucleosynthesis in inhomogeneous big bang models are considerably dependent on neutron capture reactions on light nuclei. Such reactions are also of crucial relevance for the s-process nucleosynthesis in red giant stars. To determine the reaction rates for such different temperature conditions, the neutron capture cross sections need to be known for a wide energy range.

Primordial nucleosynthesis might be affected by spatial variations of both baryon-to-photon and neutron-to-proton ratios, the later being caused by the short diffusion time for neutrons in the primordial plasma. A possible signatures of baryon-number-inhomogeneous big bang is the presence of a high primordial lithium abundance, or a high abundance of beryllium and boron isotopes. As previously mentioned, inhomogeneous big bang models involve chain reactions such as [108] ${ }^{1} \mathrm{H}(\mathrm{n}, \gamma)^{2} \mathrm{H}(\mathrm{n}, \gamma){ }^{3} \mathrm{H}(\mathrm{d}, \mathrm{n})^{4} \mathrm{He}(\mathrm{t}, \gamma)^{7} \mathrm{Li}(\mathrm{n}, \gamma)^{8} \mathrm{Li}$, and ${ }^{8} \mathrm{Li}(\alpha, \mathrm{n})^{11} \mathrm{~B}(\mathrm{n}, \gamma){ }^{12} \mathrm{~B}\left(\beta^{-}\right){ }^{12} \mathrm{C}(\mathrm{n}, \gamma){ }^{13} \mathrm{C}$, etc., paving the way to heavier nuclei. Thus, the reaction ${ }^{11} \mathrm{~B}(\mathrm{n}, \gamma){ }^{12} \mathrm{~B}$ is an important piece of inhomogeneous big bang scenarios [129].

The E1 $s$ - and $d$-wave captures to the ground state of ${ }^{12} \mathrm{~B}$ are calculated. The $J_{b}=1^{+}$ground state of ${ }^{12} \mathrm{~B}$ is described as a $j_{b}=p_{3 / 2}$ neutron coupled 
to the ${ }^{11} \mathrm{~B}$ core, which has an intrinsic spin $I_{x}=3 / 2^{-}$. Ref. [130] extracts the ground state neutron spectroscopic factors for several light by analyzing the previously reported measurements of the angular distributions in (d,p) and (p,d) reactions. We adopt the spectroscopic factor $S F=1.09$ as in Ref. [130]. Our result for the non-resonant capture (solid line) is shown in Fig. 18. The experimental data are from Ref. [131].

Similar to Ref. [131], we describe the total capture cross section by a sum of non-interfering Breit Wigner resonances superimposed on a slowly varying background (non-resonant capture, solid line in the figure) and the radiation widths of the levels are found to be $0.3 \mathrm{eV}$ at $0.36 \mathrm{MeV}, 0.3 \mathrm{eV}$ at $0.87 \mathrm{MeV}$, $0.2 \mathrm{eV}$ at $1.08 \mathrm{MeV}$, and $0.9 \mathrm{eV}$ at $1.50 \mathrm{MeV}$, with estimated uncertainties of about $50 \%$.

Without comparison to any experimental data, Ref. [132] describes a calculation using a potential model, where captures to the second and third excited states are considered. Their result is twice as large as the experimental data of Ref. [131].

In Ref. [133] the transfer reactions ${ }^{11} \mathrm{~B}(\mathrm{~d}, \mathrm{p}){ }^{12} \mathrm{~B}$ and ${ }^{12} \mathrm{C}(\mathrm{d}, \mathrm{p}){ }^{13} \mathrm{C}$, at incident energy of $11.8 \mathrm{MeV}$, have been used to extract the ANC for ${ }^{12} \mathrm{~B} \rightarrow \mathrm{n}$ $+{ }^{11} \mathrm{~B}$. The ANC found in Ref. [133] is $1.08 \mathrm{fm}^{-1 / 2}\left(C^{2}=1.16 \pm 0.10 \mathrm{fm}^{-1}\right)$. Our calculated ANC is $\sqrt{(S F) b^{2}}=1.41 \mathrm{fm}^{-1 / 2}$.

\section{5. ${ }^{12} C(n, \gamma){ }^{13} C$}

As mentioned above, not only the ${ }^{11} \mathrm{~B}(\mathrm{n}, \gamma){ }^{12} \mathrm{~B}$, but also the ${ }^{12} \mathrm{C}(\mathrm{n}, \gamma){ }^{13} \mathrm{C}$ radiative capture is an important reaction in stellar nucleosynthesis [108].

We calculated the direct capture to the ground state and the first 3 excited states of ${ }^{13} \mathrm{C}$ and compared with the experimental results of Refs. [134, 135]. The $J_{b}=1 / 2^{-}$ground state of ${ }^{13} \mathrm{C}\left(J_{b}=1 / 2^{+}\right.$for the 1 st excited state, $J_{b}=3 / 2^{-}$for the 2 nd excited state and $J_{b}=5 / 2^{+}$for the 3 rd excited state) is described as a $j_{b}=p_{1 / 2}$ neutron $\left(j_{b}=s_{1 / 2}\right.$ neutron for the 1 st excited state, $j_{b}=p_{3 / 2}$ neutron for the 2 nd excited state, $j_{b}=d_{5 / 2}$ neutron for the 3rd excited state, respectively) coupled to the ${ }^{12} \mathrm{C}$ core, which has an intrinsic spin $I_{x}=0^{+}$. In this particular case, we use $r_{0}=1.236 \mathrm{fm}, a=0.62 \mathrm{fm}$ and $V_{s o}=-7 \mathrm{MeV}$. These are the same set of parameters adopted in Ref. [136]. The spectroscopic factors published in Ref. [137] are $S F=0.77$ for the ground state, $S F=0.65$ for the 1st excited state, $S F=0.14$ for the 2nd excited state, and $S F=0.58$ for the 3 rd excited state. We adopt these values, except for the 1st excited state. For this state, we use $S F=0.8$ 
because it yields a better description of the experimental data in our model. It is also the same value adopted in Ref. [135].

It is also necessary to vary the potential depth for the continuum states for transitions to the different bound states in ${ }^{13} \mathrm{C}$. For the capture to the 1st and 3rd excited states, we use $V_{c}=V_{b}$, where $V_{b}$ are used to describe the neutron separation energies of the two excited states in ${ }^{13} \mathrm{C}$ (see Table III). For the capture to the ground state we use $V_{c}=-14.75 \mathrm{MeV}$, whereas for the capture to the $2 \mathrm{nd}$ excited state, $V_{c}=-11.50 \mathrm{MeV}$ is adopted. Our results are shown in Fig. 19. This reaction has also been studied in Refs. [136, 135, 138, 132] where a variety of potential models have been used and different spectroscopic factors were adopted.

Our calculated ANC is $\sqrt{(S F) b^{2}}=1.62 \mathrm{fm}^{-1 / 2}$ for the ground state and $1.61 \mathrm{fm}^{-1 / 2}, 0.23 \mathrm{fm}^{-1 / 2}$, and $0.11 \mathrm{fm}^{-1 / 2}$ for the 1st, 2nd and 3rd excited states, respectively. In Ref. [133] the transfer reactions ${ }^{11} \mathrm{~B}(\mathrm{~d}, \mathrm{p})^{12} \mathrm{~B}$ and ${ }^{12} \mathrm{C}(\mathrm{d}, \mathrm{p}){ }^{13} \mathrm{C}$, at incident energy of $11.8 \mathrm{MeV}$, have been used to extract the ANC for ${ }^{13} \mathrm{C} \rightarrow \mathrm{n}+{ }^{12} \mathrm{C}$. The ANC found in Ref. [132] for the 1st excited is $1.84 \pm 0.16 \mathrm{fm}^{-1 / 2}$, in close agreement with our $1.61 \mathrm{fm}^{-1 / 2}$ value.

\section{6. ${ }^{14} C(n, \gamma){ }^{15} C$}

As we have discussed previously, inhomogeneous big bang models allow for the synthesis of heavy elements via a chain of neutron capture reactions. This includes the ${ }^{14} \mathrm{C}(\mathrm{n}, \gamma){ }^{15} \mathrm{C}$ reaction. Nucleosynthesis depends on reactions that destroy ${ }^{14} \mathrm{C}$, the most important of which is ${ }^{14} \mathrm{C}(\mathrm{n}, \gamma){ }^{15} \mathrm{C}$. This reaction is also a part of the neutron induced CNO cycles in the helium burning layer of AGB stars, in the helium burning core of massive stars, and in subsequent carbon burning [139]. Such cycles may cause a depletion in the CNO abundances. The ${ }^{14} \mathrm{C}(\mathrm{n}, \gamma){ }^{15} \mathrm{C}$ reaction is the slowest of both of these cycles and, therefore the knowledge of its rate is important to predict the ${ }^{14} \mathrm{C}$ abundances.

Due to the weak binding of the ${ }^{15} \mathrm{C}$ ground state, and because there are no low lying resonances, the cross section is mainly determined by an E1 non-resonant transition from an initial p-wave scattering state to the ground state [140]. The $J_{b}=1 / 2^{+}$ground state of ${ }^{15} \mathrm{C}$ is described as a $j_{b}=s_{1 / 2}$ neutron coupled to the ${ }^{14} \mathrm{C}$ core, which has an intrinsic spin $I_{x}=0^{+}$.

In Ref. [141] a $14 \mathrm{MeV}$ deuteron beam was used to measure the angular distributions for the ${ }^{14} \mathrm{C}(\mathrm{d}, \mathrm{p}){ }^{15} \mathrm{C}$ reaction leading to the two bound states and eight of the unbound states of ${ }^{15} \mathrm{C}$. An spectroscopic factor $S F=0.88$ for the ground state of ${ }^{14} \mathrm{C}$ has been inferred. Adopting this value, we obtain 
the DC cross section shown in Fig. 21. The experimental data are from Ref. [142].

In Ref. [144] a theoretical analysis of existing experimental data on the Coulomb dissociation of ${ }^{15} \mathrm{C}$ on $208 \mathrm{~Pb}$ at $68 \mathrm{MeV} /$ nucleon was used to infer the asymptotic normalization coefficient for ${ }^{15} \mathrm{C} \rightarrow \mathrm{n}+{ }^{14} \mathrm{C}$. The $\mathrm{ANC}$ value reported in Ref. 144] is $1.13 \mathrm{fm}^{-1 / 2}\left(C^{2}=1.28 \pm 0.01 \mathrm{fm}^{-1}\right)$. Our ANC value is $\sqrt{(S F) b^{2}}=1.35 \mathrm{fm}^{-1 / 2}$.

4.7. ${ }^{15} \mathrm{~N}(n, \gamma){ }^{16} \mathrm{O}$

The cross section for the reaction ${ }^{15} \mathrm{~N}(\mathrm{n}, \gamma){ }^{16} \mathrm{~N}$ is an important input in the reaction network for the production of heavier isotopes in both inhomogeneous big bang and in red giant environments [108].

The direct capture for this reaction is dominated by the $p \rightarrow d$ wave transition to the ground state, $p \rightarrow s$ wave transition to the first excited state of ${ }^{16} \mathrm{~N}$ at $0.120 \mathrm{MeV}, p \rightarrow d$ wave transitions to the second excited state at 0.296 $\mathrm{MeV}$ and $p \rightarrow s$ wave transitions to the third excited state at $0.397 \mathrm{MeV}$. These conclusions were made in Ref. [145], where reaction cross sections of ${ }^{15} \mathrm{~N}(n, \gamma){ }^{16} \mathrm{O}$ was reported and direct capture and shell model calculations were performed to interpret their data. The gamma-ray transitions are all dominated by the $E 1$ multipolarity. The $J_{b}=2^{-}$ground state $\left(J_{b}=0^{-} 1\right.$ st excited state, $J_{b}=3^{-}$nd excited state, $J_{b}=1^{-} 3$ rd excited state) ${ }^{16} \mathrm{~N}$ is described as a $j_{b}=d_{5 / 2}$ neutron $\left(j_{b}=s_{1 / 2}\right.$ neutron, $j_{b}=d_{5 / 2}$ neutron, $j_{b}=s_{1 / 2}$ neutron) coupled to the ${ }^{15} \mathrm{~N}$ core, which has an intrinsic spin $I_{x}=1 / 2^{-}$.

In Ref. [146] $(\mathrm{d}, \mathrm{n})$ and $(\mathrm{d}, \mathrm{p})$ reactions on ${ }^{15} \mathrm{~N}$ were measured and HauserFeshbach calculations were used to extract spectroscopic factors with $30 \%$ uncertainty. Their values are $S F=0.55$ for the ground state, $S F=0.46$ for the $2^{-}$state, $S F=0.54$ for the $3^{-}$state and $S F=0.52$ for the $1^{-}$state. Our result is shown in Fig. 22, The experimental data are from Ref. [145]. Our calculations yield similar results as those of Ref. [145] and Ref. [147], and reproduce the experimental data rather well, considering the $\pm 30 \%$ error in the spectroscopic factor (see dashed line in Fig. 22).

Our calculated ANCs are $0.85 \mathrm{fm}^{-1 / 2}$ for the ground state of ${ }^{9} \mathrm{Li}, 1.10$ $\mathrm{fm}^{-1 / 2}$ for the first excited state, $0.29 \mathrm{fm}^{-1 / 2}$ for the second excited state and $1.08 \mathrm{fm}^{-1 / 2}$ for the third excited state, respectively.

\section{8. ${ }^{16} O(n, \gamma){ }^{17} \mathrm{O}$}

This reaction is important for s-processes for various metallicity stars and for inhomogeneous big bang models, which, for masses beyond $A>12$ can 
proceed via ${ }^{12} \mathrm{C}(\mathrm{n}, \gamma){ }^{13} \mathrm{C}(\mathrm{n}, \gamma){ }^{14} \mathrm{C}(\mathrm{n}, \gamma){ }^{15} \mathrm{~N}(\mathrm{n}, \gamma){ }^{16} \mathrm{~N}\left(\beta^{-}\right){ }^{16} \mathrm{O}(\mathrm{n}, \gamma) \ldots$

The non-resonant, direct capture, to the ground state and to the 1st excited state of ${ }^{17} \mathrm{O}$ dominates the cross section in the energy range of $0.02-0.28$ $\mathrm{MeV}$ [148]. The gamma-ray transitions are dominated by the E1 multipolarity and by incoming $p$-waves. The $J_{b}=5 / 2^{+}$ground state $\left(J_{b}=1 / 2^{+} 1\right.$ st excited state) of ${ }^{17} \mathrm{O}$ is described as a $j_{b}=d_{5 / 2}$ neutron $\left(j_{b}=s_{1 / 2}\right.$ neutron) coupled to the ${ }^{16} \mathrm{O}$ core, which has an intrinsic spin $I_{x}=0^{+}$. We use a spectroscopic factor $S F=1.0$ for both ground and excited states.

The results of our calculations for these two captures are shown in the top panel of Fig. 23 separately. The experimental data are from Ref. [148]. Our potential model calculations yield similar results as the calculations Ref. [149], where a microscopic multicluster model was used. The total cross section is shown in the bottom panel of Fig. 23 together with a theoretical result from Ref. [150] where direct and semi-direct components of the neutron capture cross sections were calculated.

Our calculated ANC $\left(\sqrt{(S F) b^{2}}\right)$ is $0.90 \mathrm{fm}^{-1 / 2}$ for the ground state of ${ }^{17} \mathrm{O}$ and $3.01 \mathrm{fm}^{-1 / 2}$ for the 1 st excited state of ${ }^{17} \mathrm{O}$.

\section{9. ${ }^{18} O(n, \gamma){ }^{19} \mathrm{O}$}

Further nucleosynthesis during inhomogeneous big bang models towards higher masses is controlled by the reaction rate of ${ }^{18} \mathrm{O}(\mathrm{n}, \gamma){ }^{19} \mathrm{O}$. If this reaction is stronger than the ${ }^{18} \mathrm{O}(\mathrm{p}, \alpha){ }^{15} \mathrm{~N}$ reaction then material be processed out of the CNO cycle to the region above $A>20$. This reaction is also of interest for stellar helium burning in AGB stars by means of s-processes.

The direct capture for this reaction is dominated by $p \rightarrow d$-wave transitions to the ground state, the first excited state at $0.096 \mathrm{MeV}$, and the $p \rightarrow s$ transition to the second excited state at $1.47 \mathrm{MeV}$ [151]. The gammaray transitions are all dominated by the $E 1$ multipolarity. The $J_{b}=5 / 2^{+}$ ground state $\left(J_{b}=3 / 2^{+}\right.$1st excited state, $J_{b}=1 / 2^{+} 2$ nd excited state) of ${ }^{17} \mathrm{O}$ is described as a $j_{b}=d_{5 / 2}$ neutron $\left(j_{b}=d_{3 / 2}\right.$ neutron, $j_{b}=s_{1 / 2}$ neutron $)$ coupled to the ${ }^{18} \mathrm{O}$ core, which has an intrinsic spin $I_{x}=0^{+}$.

We have adopted spectroscopic factors from Ref. [151]. They are $S F=$ 0.69 for the ground state, $S F=0.013$ for the $3 / 2+$ state, and $S F=0.83$ for the $1 / 2^{+}$state. Our results are shown in Fig. 24, They are close to the calculations reported in Refs. [151, 147]. The experimental data are from Ref. [151]. The data points at $0.138 \mathrm{MeV}$ and $0.331 \mathrm{MeV}$ are much higher than our non-resonant calculation because of the resonances at $0.152 \mathrm{MeV}$ and $0.371 \mathrm{MeV}$, corresponding to the $3 / 2^{+}$state at $4.109 \mathrm{MeV}$ and to the 
state at $4.328 \pm 003 \mathrm{MeV}$ in ${ }^{19} \mathrm{O}$, respectively. This has been discussed in details in Ref. [151].

Our calculated ANC $\left(\sqrt{(S F) b^{2}}\right)$ is $0.75 \mathrm{fm}^{-1 / 2}$ for the ground state of ${ }^{19} \mathrm{O}, 0.09 \mathrm{fm}^{-1 / 2}$ for the first excited state and $2.26 \mathrm{fm}^{-1 / 2}$ for the second excited state.

\section{Sensitivity on the potential depth parameter}

As with any other model, the results obtained with the single-particle model for the cross sections can be very sensitive to the choice of parameters. In order to check this sensitivity, in Table 7 we compare the cross sections at $0.4 \mathrm{MeV}$ for the capture to the ground state of the reaction ${ }^{16} \mathrm{O}(\mathrm{p}, \gamma){ }^{17} \mathrm{~F}$ with that of ${ }^{16} \mathrm{O}(\mathrm{n}, \gamma){ }^{17} \mathrm{O}$. The potential depth for continuum state $V_{c}$ has been varied by $\pm 10 \%$ to test the sensitivity of the cross sections on $V_{c}$.

The $V_{c}$ in the third (last) column is $10 \%$ smaller (larger) than that of the fourth column, which is used in the calculation for the S-factors or cross sections in sections III and IV. From table 7, one can conclude that proton capture is less sensitive to the internal part of the potential, as expected. This is due to the Coulomb barrier. In other words, proton capture reactions tend to be more peripheral than neutron capture reactions. In the proton capture case, the ANC technique is thus expected to work better than in the neutron capture one. But these conclusions obviously change in the presence of potential resonances, when the cross sections can suddenly change by orders of magnitude if the potential depth is slightly varied.

In order to show the large sensitivity of the S-factor, or cross section, on potential parameters close to a resonance, we use the test-case of the ${ }^{15} \mathrm{~N}(\mathrm{p}, \gamma)$ reaction. This is shown in figure 25 where we plot the ratio between the S-factor at $E=0$ calculated with a potential depth $V_{c}$ and the S-factor calculated with a zero potential depth: $S\left(0, V_{c}\right) / S(0,0)$. The open circle corresponds to the value of $V_{c}$ used in the calculation presented in figure 12 .

As is clearly seen in figure 12, a small change (i.e. by 10\%) in the value of $V_{c}$ can cause orders of magnitude change in the corresponding S-factor near a resonance. Thus, although one can indeed reproduce resonant states with the potential model, one has to be very careful with the values of observables obtained with the model, such as the ANCs, or spectroscopic factors. These will also be over-sensitive to the potential fitting parameters. 


\section{ANCs from single-particle models}

In figure 26 we show the ratio of our calculations of ANCs $\left(\sqrt{(S F) b^{2}}\right)$ with the ANCs extracted from the literature and mentioned in this article. Not all ANCs are shown because either they have not been indirectly extracted from experiments, or calculated previously. The solid circles are for proton capture whereas the solid triangles are for neutron capture. The dashed line is a guide to the eye and shows the ratio equal to unity. We notice that our ANCs differ up to a factor of 1.6 from previously reported values.

In our calculations, the ANCs are indirectly obtained by adjusting our calculated S-factors or cross sections to the available experimental data. The ANC's from literature are partially obtained by indirectly fitting calculations to experimental data in transfer reactions, or by means of elaborate microscopic models, or else. Evidently, a more consistent comparison between these values deserves a more detailed study.

\section{Final conclusions}

In this article, we have explored the single-particle potential model to describe radiative proton and neutron capture reactions of relevance for astrophysics. Using a well defined approach and the same numerical code, we have obtained spectroscopic factors and single-particle asymptotic normalization coefficients for several reactions in the mass range $A<20$.

We have only considered cases for which potential models yields reasonable results. There are several radiative capture reactions which do not fall into this category. They require a more derailed study, with possible adjustments and/or extensions of the model. Evidently, there will be situations for which the potential model will always fail.

Our work has shown minor differences with previously published results. We have demonstrated that there is a reasonable justification for the use of potential model calculations for many reactions which have either been measured experimentally, or calculated theoretically.

A systematic study of asymptotic normalization coefficients and spectroscopic factors based on the single-particle model is very useful to validate other theoretical descriptions of radiative capture reactions. This study is also relevant to correlate spectroscopic observables to other nuclear properties. Work in this direction is also in progress. 
This work was partially supported by the U.S. DOE grants DE-FG0208ER41533 and DE-FC02-07ER41457 (UNEDF, SciDAC-2).

\section{References}

[1] "Cauldrons in the Cosmos", Claus E. Rolfs and William S. Rodney, Chicago Press, Chicago 1988.

[2] C.A. Bertulani, Comput. Phys. Commun. 156 (2003) 123.

[3] K.H. Kim, M.H. Park, and B.T. Kim, Phys. Rev. C35, 363 (1987).

[4] F.C. Barker, Phys. Rev. C37, 2920 (1988).

[5] R.G.H. Robertson, Phys. Rev. C7, 543 (1973).

[6] P. Navratil, C.A. Bertulani, and E. Caurier, Phys. Lett. B 634 (2006) 191; P. Navratil, C.A. Bertulani, and E. Caurier, Phys. Rev. C73 (2006) 065801.

[7] Sofia Quaglioni and Petr Navratil, Phys. Rev. Lett. 101, 092501 (2008).

[8] H.M. Xu, C.A. Gagliardi, R.E. Tribble, A.M. Mukhamedzhanov and N.K. Timofeyuk, Phys. Rev. Lett. 73 (1994) 2027.

[9] G.M. Griffiths, E.A. Larson and L.P. Robertson, Can. J. Phys. 40, 402 (1962).

[10] B.L. Berman, L.J. Koester Jr. and J.H. Smith, Phys. Rev. 133, B117 (1964).

[11] W. Wolfli, R. Bosch, J. Lang, R. Muller, and P. Marmier, Helv. Phys. Acta 40, 946 (1967).

[12] G.J. Schmid et al, Phys. Rev. C 52, R1732 (1995).

[13] A. M. Mukhamedzhanov, R. E. Tribble, and N. K. Timofeyuk, Phys. Rev. C 51, 3472 (1995).

[14] D. Thomas, D.N. Schramm, K. A. Olive, B. D. Fields, Ap. J. 406, 569 (1993).

[15] E. Vangioni-Flam, et al., New. Astron. 4, 245 (1999). 
[16] Phys. Rev. C 56, 1144 (1997).

[17] O. Camargo et al, Phys. Rev. C 78, 034605 (2008).

[18] K. Arai et al, Nucl. Phys. A 699 (2002) 963.

[19] P. M. Prior et al, Phys. Rev. C 70, 055801 (2004).

[20] F.C. Barker, Aust. J. Phys. 33, 159 (1980).

[21] Z. E. Switkowski, J. C. P. Heggie, D. L. Kennedy, D. G. Sargood, F. C. Barker, and R. H. Spear, Nucl. Phys. A331, 50 (1980).

[22] R. Bruss et al, in: F. Käppeler, K. Wisshak (Eds.), Proc. Nuclei in the Cosmos, Karlsruhe, Germany, 1992, IOP, 1993, p. 169.

[23] Z.E. Switkowski et al, Nucl. Phys. A 331 (1979) 50.

[24] "Neutrino Astrophysics", John N. Bahcall, Cambridge University Press, 1989 ISBN: 9780521379755.

[25] D. Zahnow et al, Z. Phys. A 351229 (1995).

[26] J. M. Sampaio et al, Nucl. Phys. A 688 (2001) 518c.

[27] Q. R. Ahmad et al, Phys. Rev. Lett. 87, 071301 (2001).

[28] A. R. Junghans et al, Phys. Rev. C 68, 065803 (2003).

[29] F. J. Vaughn, R. A. Chalmers, D. Kohler and L. F. Chase, Phys. Rev. C 2, 1657 (1970).

[30] B. W. Filippone, A. J. Elwyn, C. N. Davids and D. D. Koetke, Phys. Rev.C 28, 2222 (1983).

[31] L. T. Baby et al., Phys. Rev. Lett. 90, 022501 (2003).

[32] N. Iwasa et al., Phys. Rev. Lett. 83, 2910 (1999); B. Davids et al., ibid. 86, 2750 (2001); F. Schumann et al., ibid. 90, 232501 (2003).

[33] R.W. Kavanagh et al., Bull. Am. Phys. Soc. 14, 1209 (1969).

[34] P. Descouvemont and D. Baye, Nucl. Phys. A 567, 341 (1994). 
[35] L. Trache et al, Phys. Rev. Lett. 87, 271102 (2001).

[36] P. Navratil, C. A. Bertulani and E. Caurier, Nucl. Phys. A 787, $539 \mathrm{c}(2007)$.

[52] M. Wiescher, J. Görres, S. Graff, L. Buchmann, and F.-K. Thielemann, Astrophys. J. 343, 352 (1989).

[38] G.M. Fuller, S.E. Woosley, and T.A. Weaver, Astrophys. J. 307, 675 (1986).

[39] H.M.J. Boffin, G. Paulus, M. Arnould, and N. Mowlavi, Astron. Astrophys. 279, 173 (1993).

[40] P. Mohr, Phys. Rev. C 67, 065802 (2003).

[41] L. Trache et al, Phys. Rev. C 66, 035801 (2002).

[42] D. Beaumel et al, Phys. Lett. B 514, 226 (2001).

[43] B. Guo et al, Nucl. Phys. A 761 (2005) 162.

[44] W.A. Fowler, Rev. Mod. Phys. 56, 149 (1984).

[45] K. Miura et al, Nucl. Phys. A 539, 441 (1992).

[46] S. Cohen and D. Kurath, Nucl. Phys. A101, 1 (1967).

[47] S. Varma and P. Goldhammer, Nucl . Phys. A125, 193 (1969).

[48] D. Zahnow, C. Angulo, M. Junker, C. Rolfs, S. Schmidt, W. H. Schulte and E. Somorjai, Nucl. Phys. A 589, 95 (1995).

[49] A. Sattarov et al, Phys. Rev. C 60, 035801 (1999).

[50] F. C. Barker, Nucl. Phys. A 697 (2002) 915.

[51] A. M. Mukhamedzhanov et al, Phys. Rev. C 56, 1302 (1997).

[52] M. Wiescher et al., Astrophys. J. 343, 352 (1989).

[53] Xiaodong Tang et al, Phys. Rev. C 67015804 (2003).

[54] A. Lefebvre et al., Nucl. Phys. A 592, 69 (1995). 
[55] R.E. Tribble et al, Nucl. Phys. A 718 (2003) 147c.

[56] Weiping Liu et al, Nucl. Phys. A 728 (2003) 275.

[57] C. D. Nesaraja et al, Phys. Rev. C 64, 065804 (2001).

[58] C. Rolfs and R. E. Azuma, Nucl. Phys. A227, 291 (1974).

[59] C. Mahaux, Nucl. Phys. 71 (1965) 241.

[60] N. Burtebaev et al, Phys. Rev. C 78, 035802 (2008).

[61] K. Langanke et al, Nucl. Phys. A 435 (1985) 657.

[62] R. Yarmukhamedov, Yad. Fiz. 60, 1017 (1997).

[63] S. V. Artemov et al, Izv. RAN (Bull. Russ. Acad. Sci.) Ser. Fiz. 66, 60 (2002).

[64] R.K. Ulrich, "Essays in Nuclear Astrophysics", Edited by C.A. Barnes, D.D. Clayton, D.N. Schramm, Cambridge Univ. Press, Cambridge, UK, 1982, p. 301.

[65] M. Lugaro, F. Herwig, J.C. Lattanzio, R. Gallino, O. Straniero, Astrophys. J. 586, 1305 (2003).

[66] J. D. King et al, Nucl. Phys. A567, 354 (1994).

[67] A.M. Mukhamedzhanov et al, Nucl. Phys. A 725 (2003) 279.

[68] Guo Bing, Li Zhi-hong, Chin. Phys. Lett. 24, 65 (2007).

[69] Z. H. Li et al, Phys. Rev. C 74, 035801 (2006).

[70] Th. Delbar et al, Phys. Rev. C 48, 3088 (1993).

[71] Xiaodong Tang et al, Phys. Rev. C 69, 055807 (2004).

[72] P. Decrock et al, Phys. Rev. C 48, 2057 (1993).

[73] P. Descouvemont and D. Baye, Nucl. Phys. A500, 155 (1989).

[74] S. Ahlen et al., Phys. Lett. B249, 149 (1990). 
[75] C. Arpesella et al., Phys. Lett. B658, 101 (2008).

[76] G. Imbriani et al., Eur. Phys. J. A 25, 455 (2005).

[77] Schurmann et al, Phys. Rev. C 77, 055803 (2008).

[78] A. Formicola et al, Phys. Lett. B 591 (2004) 61

[79] S. O. Nelson et al, Phys. Rev. C 68, 065804 (2003).

[80] S. Raman et al., Phys. Rev. C 50, 682 (1994).

[81] P. F. Bertone et al, Phys. Rev. C 66, 055804 (2002).

[82] R. E. Pixley. The reaction cross section of nitrogen 14 for protons between $220 \mathrm{keV}$ and $600 \mathrm{keV}$. Ph. D. Thesis, California Institute of Technology, 1957.

[83] U. Schroeder et al, Nucl. Phys. A467 (1987) 240.

[84] C. Angulo et al, Nucl. Phys. A 758 (2005) 391c.

[85] A. M. Mukhamedzhanov et al, Phys. Rev. C 67, 065804 (2003)

[86] C. Rolfs, W. S. Rodney, Nucl. Phys. A 235 (1974) 450.

[87] H. W. Fulbright, J. A. Robins, M. Blann, D. G. Fleming, and H. S. Plendl, Phys. Rev. 184, 1068 (1969).

[88] A. M. Mukhamedzhanov, P. Bem, V. Burjan et al, Phys. Rev. C 78, 015804 (2008).

[89] F. Herwig, Annu. Rev. Astron. Astrophys. 43, 435 (2005).

[90] "Asymptotic Giant Branch Stars", H. J. Habing and H. Olofsson, (Springer, Heidelberg, 2004).

[91] D. F. Hebbard, Nucl. Phys. 15 (1960) 289.

[92] C.A. Bertulani, P. Danielewicz, Nucl. Phys. A 717199 (2003).

[93] C. Rolfs, Nucl. Phys. A 217 (1973) 29 
[94] R. E. Hester, R. E. Pixley and W. A. Lamb, Phys. Rev. 1111604 (1958).

[95] N. Tanner, Phys. Rev. 1141060 (1959).

[96] R. Morlock, R. Kunz, A. Mayer et al, Phys. Rev. Lett. 793837 (1997).

[97] K. Bennaceur et al, Phys. Lett. B 488, 75 (2000).

[98] C. A. Gagliardi et al., Phys. Rev. C 59, 1149 (1999).

[99] J. B. Marion and W. A. Fowler, Astrophys. J. 125, 221 (1957).

[100] C. Rolfs, W.S. Rodney, M.H. Shapiro, and H. Winkler, Nucl. Phys. A 241, 460 (1975).

[101] A. M. Mukhamedzhanov et al, Phys. Rev. C 73, 035806 (2006).

[102] P. J. E. Peebles, Phys. Rev. Lett. 16, 410 (1966).

[103] H. Sato, Prog. Theor. Phys. 38, 1083 (1967).

[104] R. V.Wagoner,W. A. Fowler, and F. Hoyle, Astrophys. J. 148, 3 (1967).

[105] S. W. Stahler, Astrophys. J. 332, 804 (1988).

[106] C. Casella et al., Nucl. Phys. A706, 203 (2002).

[107] J. H. Applegate and C. J. Hogan, Phys. Rev. D 31, 3037 (1985).

[108] J. H. Applegate, C. J. Hogan, and R. J. Scherrer, Phys. Rev. D 35, 1151 (1987); Ap. J. 329, 572 (1988).

[109] R. A. Malaney and W. A. Fowler, Astrophys. J. 333, 14 (1988).

[110] Y. Nagai et al, Phys. Rev. C 74, 025804 (2006).

[111] R. B. Wiringa, V. G. J. Stoks, and R. Schiavilla, Phys. Rev. C 51, 38 (1995).

[112] B. S. Pudliner, V. R. Pandharipande, J. Carlson, S. C. Pieper, and R. B. Wiringa, Phys. Rev. C 56, 1720 (1997).

[113] B.W. Filippone et al., Phys. Rev. Lett. 50, 452 (1983). 
[114] G.M. Fuller, G.J. Mathews and C.R. Alcock, Phys. Rev. D 37, 1380 (1988).

[115] Y. Nagai, et al., Phys. Rev. C 71, 055803 (2005).

[116] W. L. Imhof et al., Phys. Rev. 114, 1037 (1959).

[117] M.Wiescher, R. Steininger, and F. Kaeppeler, Astrophys. J. 344, 464 (1989).

[118] Y. Nagai et al, Astrophys. J. 381, 444 (1991).

[119] M. Heil, F. Kaeppeler, M.Wiescher, and A. Mengoni, Astrophys. J. 507, 997 (1998).

[120] J.E. Lynn, E.T. Jurney and S. Raman, Phys. Rev. 44, 764 (1991).

[121] S. E. Wooseley, J. R. Wilson, G. J. Mathews, R. D. Hoffman, and B. S. Meyer, Astrophys. J. 433, 229 (1994).

[122] V. D. Efros, W. Balogh, H. Herndl, R. Hofinger, and H. Oberhummer, Z. Phys. A 355, 101 (1996).

[123] S. K. Rosswog, C. Friburghaus, and F.-K. Thielemann, Nucl. Phys. A688, 344 (2001).

[124] C. A. Bertulani, J. Phys. G 25, (1999) 1959.

[125] Z.Q. Mao and A.E. Champagne, Nucl. Phys. A 522 (1991) 568.

[126] R. Kanungo et al, Phys. Lett. B 660 (2008) 26.

[127] P. D. Zecher, A. Galonsky, S. Gaff et al, Phys. Rev. C 57, (1998) 959.

[128] P. Banerjee, R. Chatterjee and R. Shyam, Phys. Rev. C 78, 035804 (2008).

[129] T. Kajino, G .J. Mathews, and G. M. Fuller, Astrophys. J. 364, 7 (1990).

[130] M. B. Tsang, Jenny Lee and W. G. Lynch, Phys. Rev. Lett. 95, 222501 (2005). 
[131] W. L. Imhof, R. G. Johnson, F. J. Vaughn, and M. Walt, Phys. Rev. 125, 1334 (1962).

[132] C. J. Lin et al, Phys. Rev. C 68, 047601 (2003).

[133] Z. H. Liu et al, Phys. Rev. C 64, 034312 (2001).

[134] T. Ohsaki, Y. Nagai, M. Igashira, T. Shima, K. Takeda, S. Seino, and T. Irie, Astrophys. J. 422, 912 (1994).

[135] T. Kikuchi et al, Phys. Rev. C 57, 2724 (1998).

[136] A. Mengoni et al, Phys. Rev. C 52, R2334 (1995).

[137] F. Ajzenberg-Selove, Nucl. Phys. A 523, 1 (1991).

[138] D. Baye, Phys. Rev. C 70, 015801 (2004).

[139] M. Wiescher, J. Görres, and H. Schatz, J. Phys. G 25, R133 (1999).

[140] N. K. Timofeyuk et al, Phys. Rev. Lett. 96, 162501 (2006).

[141] J. D. Goss et al., Phys. Rev. C 12, 1730 (1975).

[142] R. Reifarth et al, Phys. Rev. C 77 015804(2008).

[143] M. Wiescher, J. Gorres and F. K. Thielemann, The Astrophysical Journal 363, 340 (1990).

[144] N. C. Summers, F. M. Nunes, Phys. Rev. C 78, 011601(R) (2008).

[145] J. Meissner et al, Phys. Rev. C 53, 977 (1996).

[146] W. Bohne et al, Nucl. Phys. A 196, 41 (1972).

[147] H. Herndl et al, Phys. Rev. C 60, 064614 (1999).

[148] M. Igashira, Y. Nagai, K. Masuda, T. Ohsaki, and H. Kitazawa, Astrophys. J. 441, L89 (1995).

[149] M. Dufour, P. Descouvemont, Nucl. Phys. A 694 (2001) 221.

[150] S. Chiba, H. Koura, T. Hayakawa et al, Phys. Rev. C 77, 015809 (2008)

[151] J. Meissner et al, Phys. Rev. C 53, 459 (1996). 


\section{TABLES AND GRAPHS}

\begin{tabular}{|c|c|}
\hline Parameter & Adopted value \\
\hline$R_{0}=R_{S 0}=R_{C}$ & $r_{0}(A+1)^{1 / 3} \mathrm{fm}$ \\
\hline$r_{0}$ & 1.25 \\
\hline$a_{0}=a_{S 0}$ & $0.65 \mathrm{fm}$ \\
\hline$V_{s 0}$ & $-10 \mathrm{MeV}$ \\
\hline
\end{tabular}

Table 1: Parameters of the single-particle potentials, except for few cases explicitly mentioned in the text. 


\begin{tabular}{|c|c|c|c|c|c|c|}
\hline Reaction & $E_{b}$ & $V_{b}$ & $S F$ & $b$ & $>R_{0}$ & $S(0)$ \\
\hline $\mathrm{d}(\mathrm{p}, \gamma){ }^{3} \mathrm{He}$ & 5.49 & -44.43 & 0.7 & 1.86 & 0.98 & 0.14 \\
\hline${ }^{6} \mathrm{Li}(\mathrm{p}, \gamma){ }^{7} \mathrm{Be}$ & 5.61 & -65.91 & $0.83[17]$ & 2.21 & 1.28 & 66.8 \\
\hline${ }^{6} \mathrm{Li}(\mathrm{p}, \gamma)^{7} \mathrm{Be}^{*}$ & 5.18 & -64.94 & $0.84[18]$ & 2.08 & 1.19 & 32.7 \\
\hline${ }^{7} \mathrm{Li}(\mathrm{p}, \gamma){ }^{8} \mathrm{Be}$ & 17.26 & -75.69 & 1.0 & 7.84 & 1.01 & 238. \\
\hline${ }^{7} \mathrm{Be}(\mathrm{p}, \gamma)^{8} \mathrm{~B}$ & 0.14 & -41.26 & 1.0 & 0.72 & 1.00 & 19.4 \\
\hline${ }^{8} \mathrm{~B}(\mathrm{p}, \gamma){ }^{9} \mathrm{C}$ & 1.30 & -41.97 & $1.0[40]$ & 1.31 & 1.08 & 42.5 \\
\hline${ }^{9} \mathrm{Be}(\mathrm{p}, \gamma){ }^{10} \mathrm{~B}$ & 6.59 & -49.83 & $1.0[45]$ & 3.43 & 1.27 & 1052 \\
\hline${ }^{11} \mathrm{C}(\mathrm{p}, \gamma){ }^{12} \mathrm{~N}$ & 0.60 & -40.72 & $0.4[54]$ & 1.49 & 1.01 & 50.8 \\
\hline${ }^{12} \mathrm{C}(\mathrm{p}, \gamma){ }^{13} \mathrm{~N}$ & 1.94 & -41.65 & 1.0 & 2.05 & 1.04 & 2346 \\
\hline${ }^{13} \mathrm{C}(\mathrm{p}, \gamma)^{14} \mathrm{~N}$ & 7.55 & -50.26 & 0.33 & 5.31 & 1.10 & 6217 \\
\hline${ }^{13} \mathrm{~N}(\mathrm{p}, \gamma)^{14} \mathrm{O}$ & 4.63 & -46.02 & $1.88[69]$ & 3.97 & 1.45 & 5771 \\
\hline${ }^{14} \mathrm{~N}(\mathrm{p}, \gamma)^{15} \mathrm{O}^{*}$ & 0.50 & -14.83 & 1.5 & 4.24 & 1.00 & 1470 \\
\hline${ }^{15} \mathrm{~N}(\mathrm{p}, \gamma){ }^{16} \mathrm{O}$ & 12.13 & -54.81 & $1.8[86]$ & 10.16 & 0.78 & $2.21 \cdot 10^{4}$ \\
\hline${ }^{16} \mathrm{O}(\mathrm{p}, \gamma)^{17} \mathrm{~F}$ & 0.60 & -49.69 & $0.9[93]$ & 0.96 & 1.02 & 304 \\
\hline${ }^{16} \mathrm{O}(\mathrm{p}, \gamma){ }^{17} \mathrm{~F}^{*}$ & 0.11 & -50.70 & $1.0[93]$ & 77.21 & 1.00 & 9075 \\
\hline${ }^{20} \mathrm{Ne}(\mathrm{p}, \gamma)^{21} \mathrm{Na}^{*}$ & 0.006 & -47.24 & 0.7 & 4.02 & 1.00 & $4.28 \cdot 10^{4}$ \\
\hline${ }^{20} \mathrm{Ne}(\mathrm{p}, \gamma)^{21} \mathrm{Na}^{*}$ & 2.10 & -49.63 & 0.8 & 2.43 & 1.00 & 2493 \\
\hline
\end{tabular}

Table 2: Binding energy $\left(E_{b}\right.$, in $\left.\mathrm{MeV}\right)$, central potential depth of bound state $\left(V_{b}\right.$, in $\mathrm{MeV})$, spectroscopic factor $(S F)$, single-particle asymptotic normalization coefficients $(b$, in $\mathrm{fm}^{-1 / 2}$ ), the factor that multiplies S-factor if the integration in Eq. 6 starts at $r=R_{0}$ (nuclear radius) and S-factor at zero energy $(S(0)$, in $\mathrm{eV}$ b) for radiative proton capture reactions. 


\begin{tabular}{|c|c|c|c|c|c|}
\hline Reaction & $\overline{E_{b}}$ & $V_{b}$ & $S F$ & $b$ & $r>R_{0}$ \\
\hline${ }^{2} \mathrm{H}(\mathrm{n}, \gamma){ }^{3} \mathrm{H}$ & 6.26 & -44.63 & 1.0 & 1.90 & 0.97 \\
\hline${ }^{7} \mathrm{Li}(\mathrm{n}, \gamma){ }^{8} \mathrm{Li}$ & 2.03 & -43.56 & $0.87[115]$ & 0.76 & 1.04 \\
\hline${ }^{7} \mathrm{Li}(\mathrm{n}, \gamma)^{8} \mathrm{Li}^{*}$ & 1.05 & -40.46 & $0.48[115]$ & 0.47 & 1.02 \\
\hline${ }^{8} \mathrm{Li}(\mathrm{n}, \gamma){ }^{9} \mathrm{Li}$ & 4.06 & -45.29 & $0.8[126]$ & 1.25 & 1.08 \\
\hline${ }^{8} \mathrm{Li}(\mathrm{n}, \gamma){ }^{9} \mathrm{Li}^{*}$ & 1.37 & -38.57 & $0.55[125]$ & 0.54 & 1.03 \\
\hline${ }^{11} \mathrm{~B}(\mathrm{n}, \gamma){ }^{12} \mathrm{~B}$ & 3.37 & -34.33 & $1.09[130]$ & 1.35 & 1.09 \\
\hline${ }^{12} \mathrm{C}(\mathrm{n}, \gamma){ }^{13} \mathrm{C}$ & 4.95 & -41.35 & $0.77[137]$ & 1.85 & 3.23 \\
\hline${ }^{12} \mathrm{C}(\mathrm{n}, \gamma){ }^{13} \mathrm{C}^{*}$ & 1.86 & -56.90 & $0.8[135]$ & 1.80 & 1.00 \\
\hline${ }^{12} \mathrm{C}(\mathrm{n}, \gamma){ }^{13} \mathrm{C}^{*}$ & 1.27 & -28.81 & 0.14 [137] & 0.61 & 1.23 \\
\hline${ }^{12} \mathrm{C}(\mathrm{n}, \gamma){ }^{13} \mathrm{C}^{*}$ & 1.09 & -56.85 & 0.58 [137] & 0.15 & 1.04 \\
\hline${ }^{14} \mathrm{C}(\mathrm{n}, \gamma){ }^{15} \mathrm{C}$ & 1.22 & -48.63 & 0.88 [141] & 1.44 & 1.00 \\
\hline${ }^{15} \mathrm{~N}(\mathrm{n}, \gamma){ }^{16} \mathrm{~N}$ & 2.49 & -27.06 & $0.55[146]$ & 1.14 & 1.38 \\
\hline${ }^{15} \mathrm{~N}(\mathrm{n}, \gamma){ }^{16} \mathrm{~N}^{*}$ & 2.37 & -12.45 & $0.46[146]$ & 1.62 & 1.11 \\
\hline${ }^{15} \mathrm{~N}(\mathrm{n}, \gamma){ }^{16} \mathrm{~N}^{*}$ & 2.19 & -49.51 & $0.54[146]$ & 0.39 & 2.77 \\
\hline${ }^{15} \mathrm{~N}(\mathrm{n}, \gamma){ }^{16} \mathrm{~N}^{*}$ & 2.09 & -11.90 & $0.52[146]$ & 1.50 & 0.94 \\
\hline${ }^{16} \mathrm{O}(\mathrm{n}, \gamma){ }^{17} \mathrm{O}$ & 4.14 & -51.77 & 1.0 & 0.90 & 1.17 \\
\hline${ }^{16} \mathrm{O}(\mathrm{n}, \gamma){ }^{17} \mathrm{O}^{*}$ & 3.27 & 51.60 & 1.0 & 3.01 & 0.99 \\
\hline${ }^{18} \mathrm{O}(\mathrm{n}, \gamma){ }^{19} \mathrm{O}$ & 3.96 & -47.79 & $0.69[151]$ & 0.90 & 1.17 \\
\hline${ }^{18} \mathrm{O}(\mathrm{n}, \gamma){ }^{19} \mathrm{O}^{*}$ & 3.86 & -55.94 & $0.013[151]$ & 0.81 & 1.14 \\
\hline${ }^{18} \mathrm{O}(\mathrm{n}, \gamma){ }^{19} \mathrm{O}^{*}$ & 2.49 & -46.33 & $0.83[151]$ & 2.48 & 1.00 \\
\hline
\end{tabular}

Table 3: Binding energy $\left(E_{b}\right.$, in $\left.\mathrm{MeV}\right)$, central potential depth of bound state $\left(V_{b}\right.$, in $\mathrm{MeV})$, spectroscopic factor $(S F)$, single-particle asymptotic normalization coefficients $(b$, in $\mathrm{fm}^{-1 / 2}$ ) and the factor multiplying the S-factor assuming that the integration in Eq. 6 starts at $r=R_{0}$ (nuclear radius).

\begin{tabular}{|c|c|c|c|c|}
\hline \multirow{2}{*}{${ }^{16} \mathrm{O}(\mathrm{p}, \gamma){ }^{17} \mathrm{~F}$} & $V_{c}(\mathrm{MeV})$ & 44.72 & 49.69 & 54.66 \\
\cline { 2 - 5 } & $\sigma(\mu b)$ & $4.63 \times 10^{-3}$ & $4.83 \times 10^{-3}$ & $5.05 \times 10^{-3}$ \\
\cline { 2 - 5 } & $\Delta \sigma / \sigma$ & $-4.14 \%$ & & $+4.55 \%$ \\
\hline \multirow{3}{*}{${ }^{16} \mathrm{O}(\mathrm{n}, \gamma){ }^{17} \mathrm{O}$} & $V_{c}(\mathrm{MeV})$ & 46.59 & 51.77 & 56.94 \\
\cline { 2 - 5 } & $\sigma(\mu b)$ & 14.35 & 21.41 & 38.42 \\
\cline { 2 - 5 } & $\Delta \sigma / \sigma$ & $-32.98 \%$ & & $+79.45 \%$ \\
\hline
\end{tabular}

Table 4: Cross sections at $0.4 \mathrm{MeV}$ for the capture to the ground state of the reaction ${ }^{16} \mathrm{O}(\mathrm{p}, \gamma){ }^{17} \mathrm{~F}$ with that of ${ }^{16} \mathrm{O}(\mathrm{n}, \gamma){ }^{17} \mathrm{O}$. 


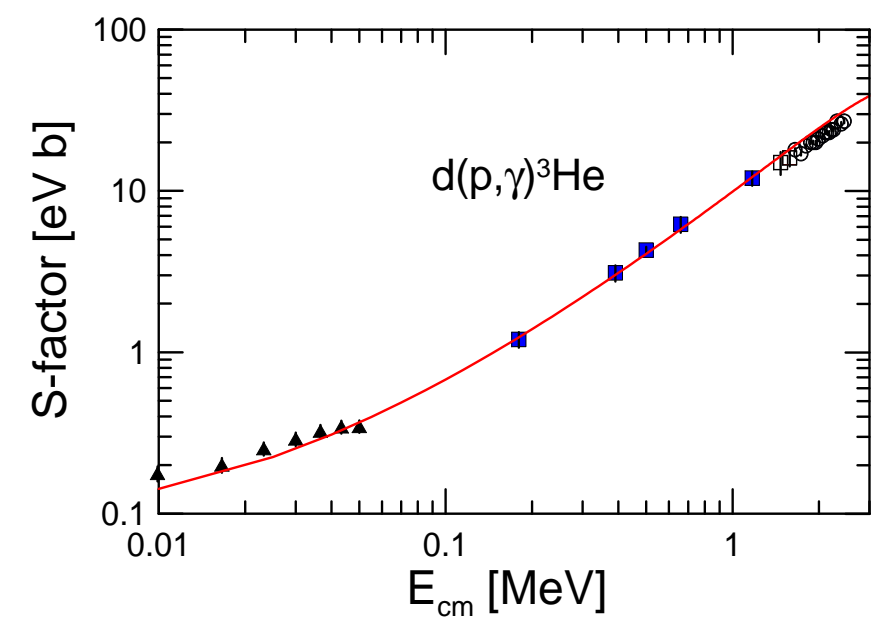

Figure 1: (Color online). Single-particle model calculation for the reaction $\mathrm{d}(p, \gamma)^{3} \mathrm{He}$. Experimental data are from Refs. 9, 10, 11, 12]. The parameters calculated according to Table I are used. The potential depth (here $V_{b}=V_{c}$ ) is given in Table II.

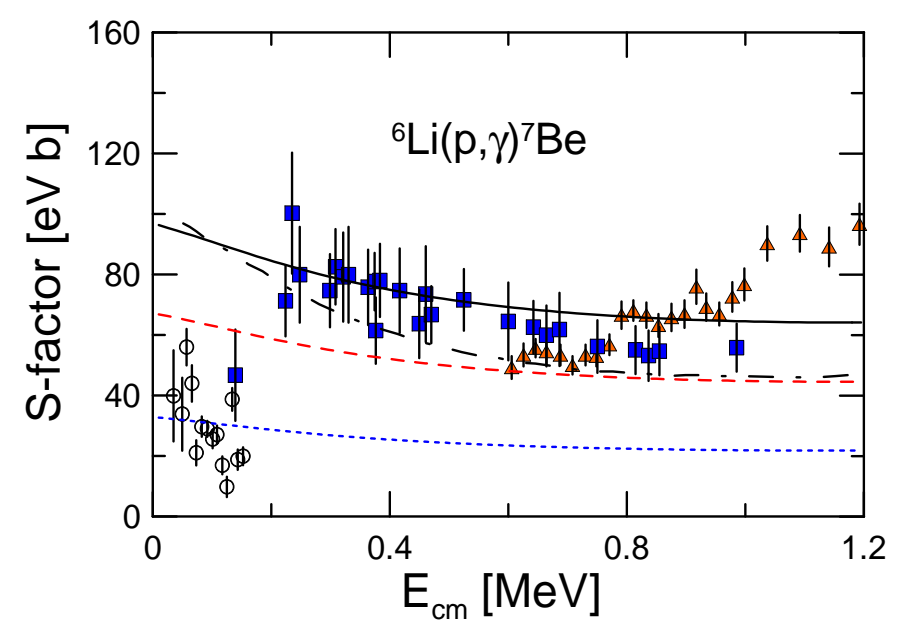

Figure 2: (Color online). Single-particle model calculation for the reaction ${ }^{6} \mathrm{Li}(\mathrm{p}, \gamma){ }^{7} \mathrm{Be}$. The dotted line is the calculation for the capture to the 1 st excited of ${ }^{7} \mathrm{Be}$ and the dashed line for the ground state. The solid line is the total calculated S-factor. Experimental data are from Refs. 22, 23, 18]. The dotted-dashed line is the total S-factor calculated in Ref. [18] using a four-cluster microscopic model. 


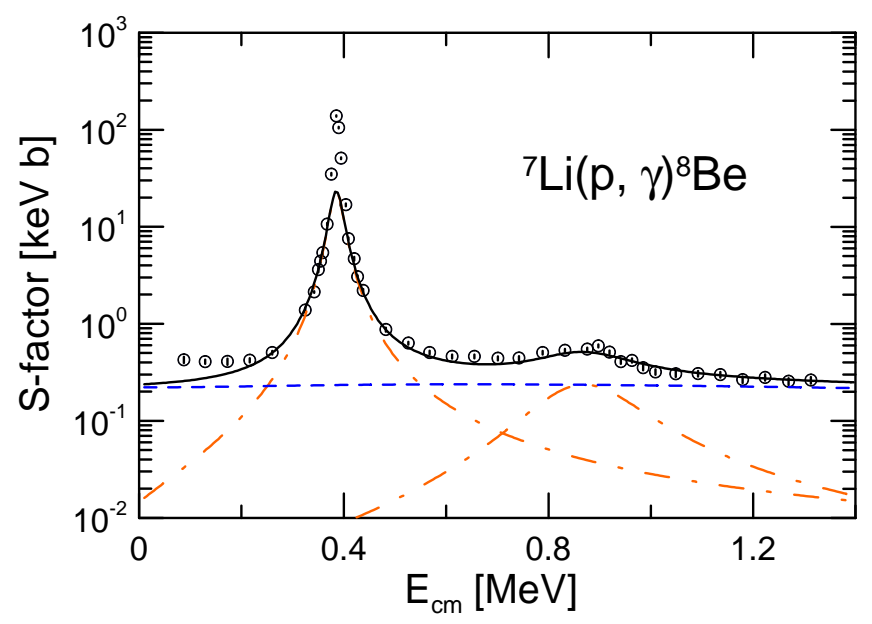

Figure 3: (Color online). Potential model calculation for the reaction ${ }^{7} \mathrm{Li}(\mathrm{p}, \gamma){ }^{8} \mathrm{Be}$. Experimental data are from Ref. [25].

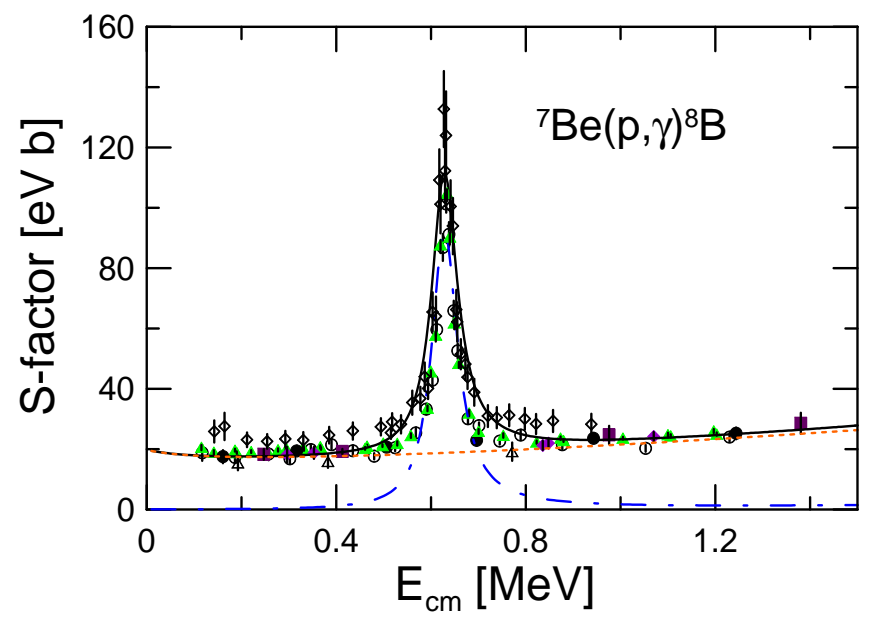

Figure 4: (Color online). Single-particle model calculations for the reaction ${ }^{7} \mathrm{Be}(p, \gamma)^{8} \mathrm{~B}$. The dashed-dotted line is the calculation for the M1 resonance at $E_{c m}=0.63 \mathrm{MeV}$ and the dotted line is for the non-resonant capture. Experimental data are from Refs. 29, 30, 31, 28, 32, 33]. The total S factor is shown as a solid line. 


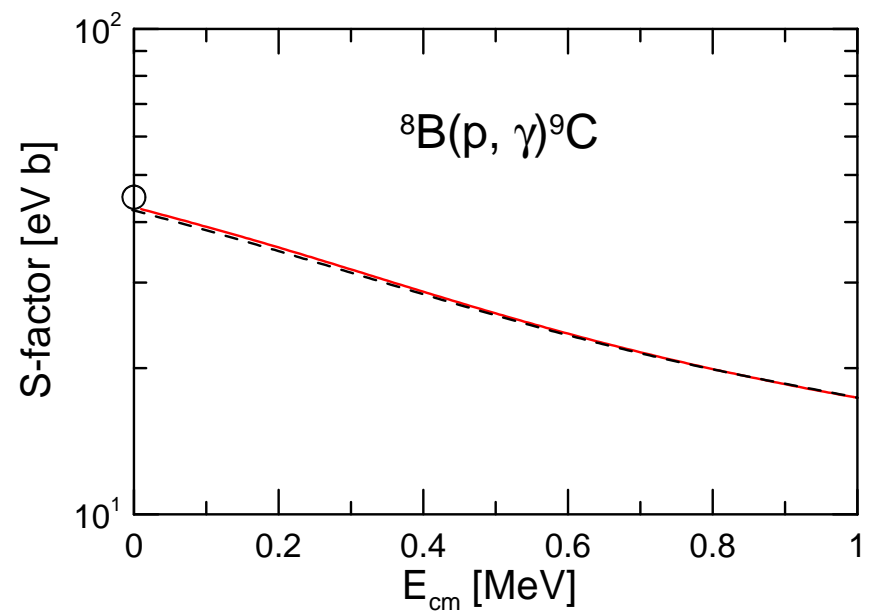

Figure 5: (Color online). Single-particle model calculations for the reaction ${ }^{8} \mathrm{~B}(\mathrm{p}, \gamma)^{9} \mathrm{C}$ (solid line). The open circle at $E=0$ is from Refs. [41, 42]. The result from Ref. 40] $\left(\lambda_{\text {scatt }}=0.55 \mathrm{fm}\right)$ is shown as a dashed line.

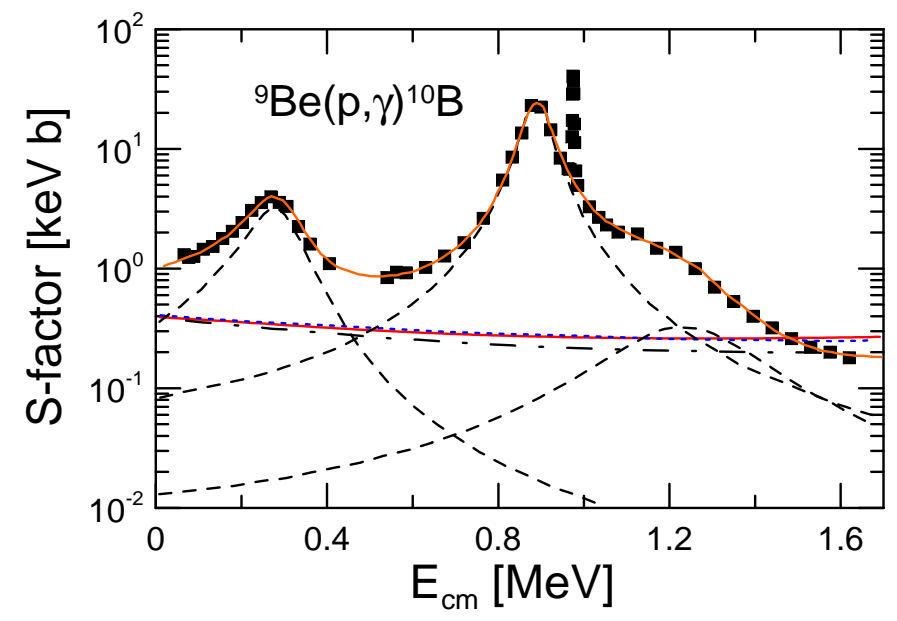

Figure 6: (Color online). Single-particle model calculations for the reaction ${ }^{9} \mathrm{Be}(\mathrm{p}, \gamma){ }^{10} \mathrm{~B}$ (solid line). The experimental data are from Ref. [48]. The fits to the resonances, done in Ref. [48], are shown as dashed lines. DC results from Ref. [49] and Ref. [48] are shown as a dotted-dashed line and a dotted line, respectively. The curve passing through the experimental data points is the sum of our DC calculation and the resonance fits, given by the dashed lines. 


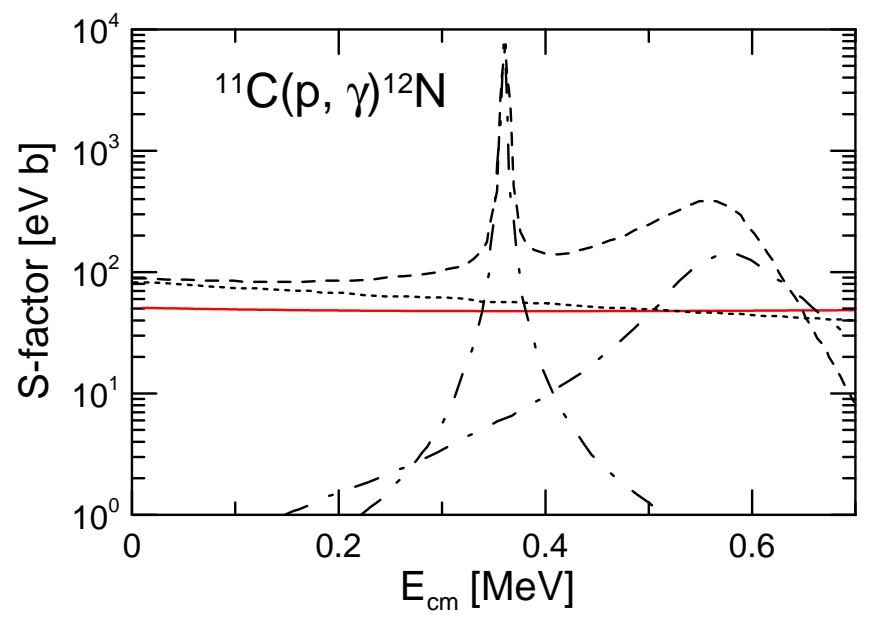

Figure 7: (Color online). Single-particle model calculations for the reaction ${ }^{11} \mathrm{C}(\mathrm{p}, \gamma){ }^{12} \mathrm{~N}$ (solid line). R-matrix results from Ref. [53] are also shown by dashed lines (resonances) and a dotted line (non-resonant).

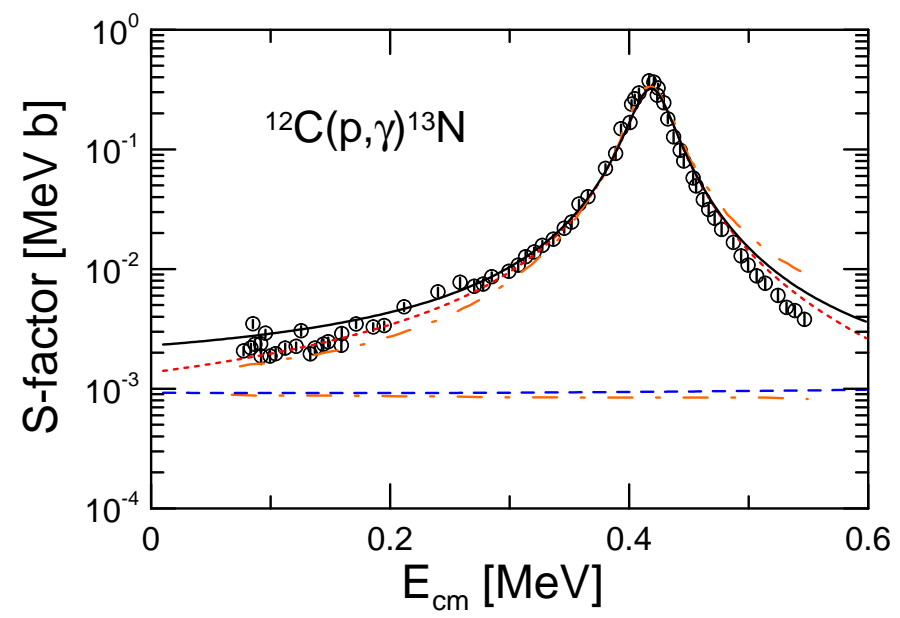

Figure 8: (Color online). Single-particle model calculations for the reaction ${ }^{12} \mathrm{C}(\mathrm{p}, \gamma){ }^{13} \mathrm{~N}$ are shown as a dashed line (DC), a dotted line ( $E 1$ resonance) and a solid line (total). The experimental data are from Ref. [58]. The potential model results from Ref. [57] are shown as dotted-dashed lines. 


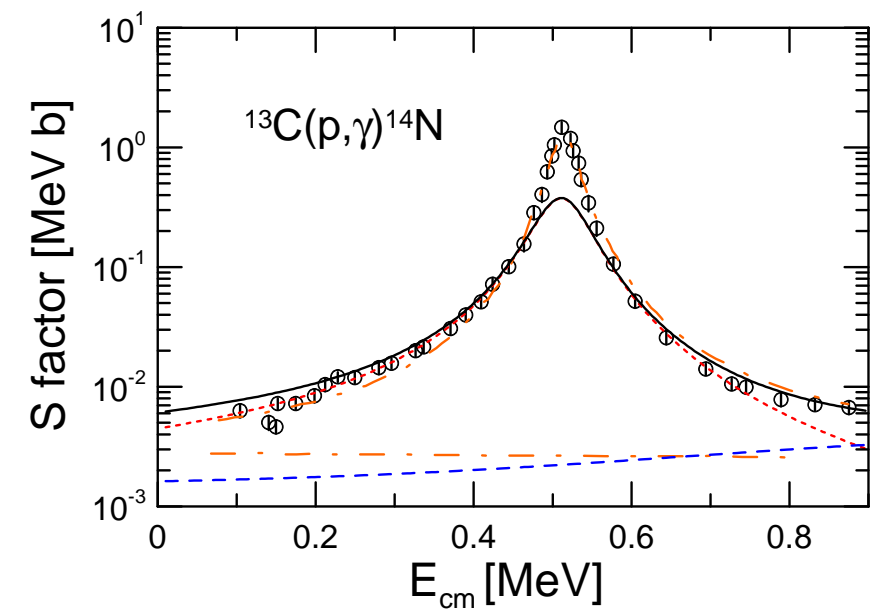

Figure 9: (Color online). Single-particle model calculations for the reaction ${ }^{13} \mathrm{C}(\mathrm{p}, \gamma){ }^{14} \mathrm{~N}$ are shown as a dashed line (DC), a dotted line (E1 resonance) and a solid line (total). The experimental data are from Ref. [66]. The potential model results from Ref. [57] are shown as dotted-dashed lines.

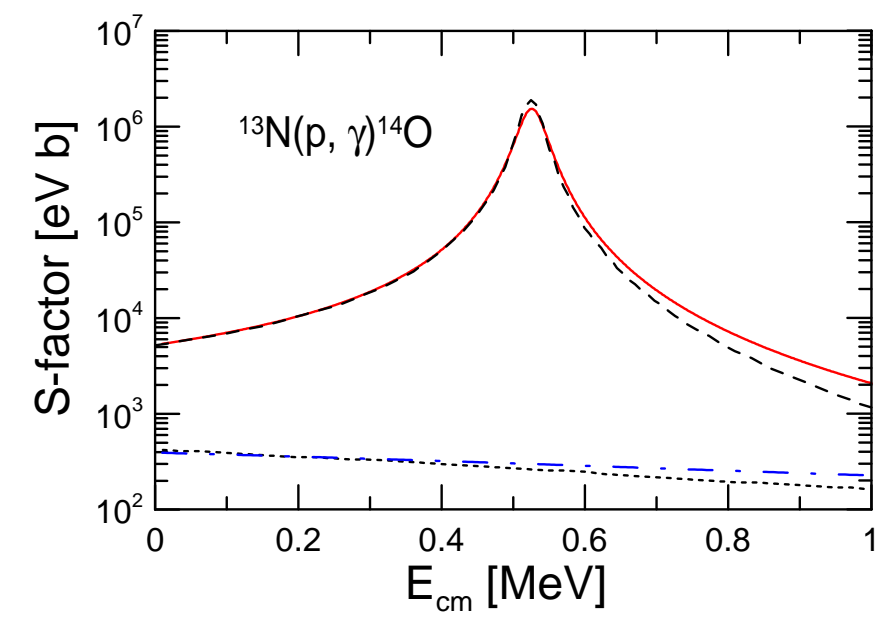

Figure 10: (Color online). Single-particle model calculations for the reaction ${ }^{13} \mathrm{~N}(\mathrm{p}, \gamma){ }^{14} \mathrm{O}$ are shown as a dotted-dashed line (non-resonant) and a solid line (E1 resonance). Rmatrix results from Ref. 68] are also shown as a dashed line (resonance) and a dotted line (non-resonant). 


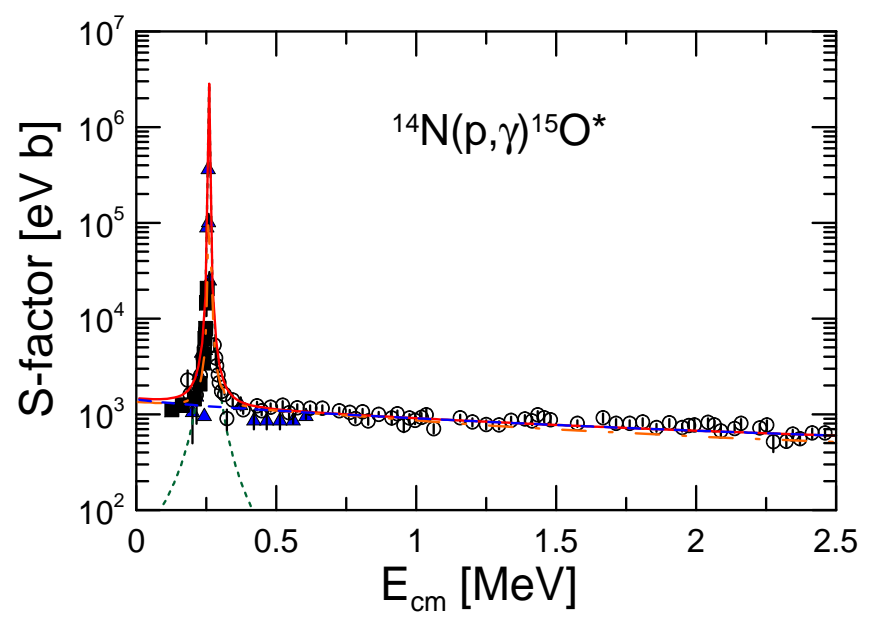

Figure 11: (Color online). Single-particle model calculations for ${ }^{14} \mathrm{~N}(\mathrm{p}, \gamma){ }^{15} \mathrm{O}$ capture to the $6.793 \mathrm{MeV}$ excited state of ${ }^{15} \mathrm{O}$. Dashed line is for the non-resonat capture, dotted line is for the M1 resonance, and the solid line is the total S-factor. The experimental data are from Refs. 78, 82, 83]. The dotted-dashed line is a R-matrix fit obtained in Ref. [78] with the channel radius $a=5.5 \mathrm{fm}$ (this curve is almost invisible because it is very close to our results).

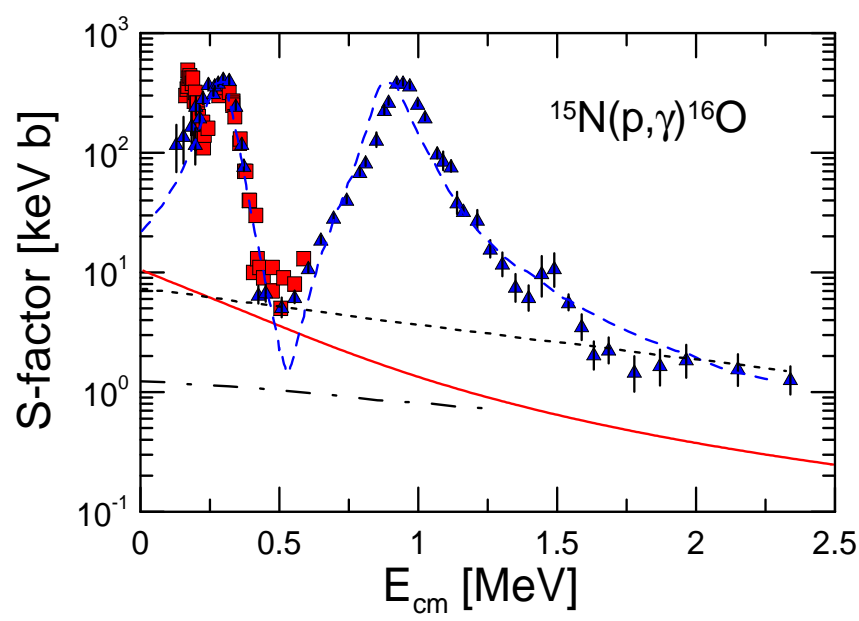

Figure 12: (Color online). Single-particle model calculation for the reaction ${ }^{15} \mathrm{~N}(\mathrm{p}, \gamma){ }^{16} \mathrm{O}$ (solid line). The experimental data are from Refs. [86, 91]. Dashed lines are Breit-Wigner fits to the resonances, as described in Ref. [86]. The dotted line is a non-resonant capture of Ref. 86]. The dotted-dashed line represents the non-resonant capture calculation from Ref. [88]. 


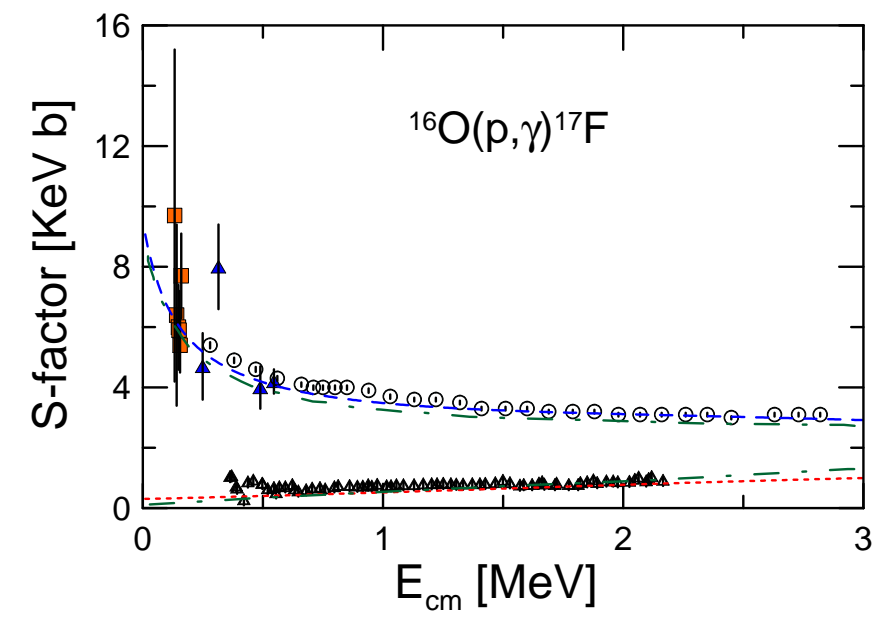

Figure 13: (Color online). Single-particle model calculation for the reaction ${ }^{16} \mathrm{O}(\mathrm{p}, \gamma){ }^{17} \mathrm{~F}$. The dotted line and the dashed line are for the capture to the ground state and to the first excited state respectively. The experimental data are from Refs. [94, 95, 93, 96]. The dotted-dashed lines are the result of shell model calculations published in Ref. [97].

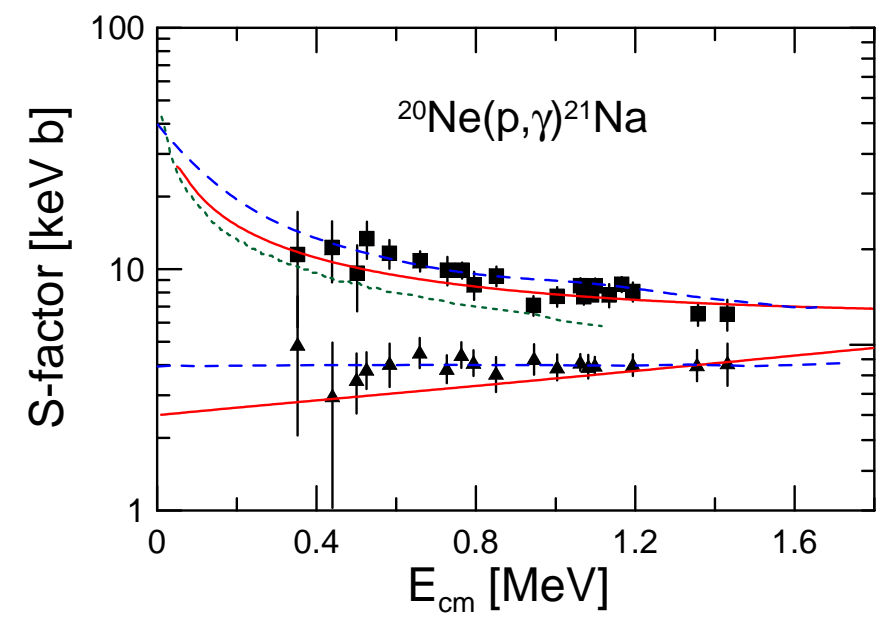

Figure 14: (Color online). Single-particle model calculation for the reaction ${ }^{20} \mathrm{Ne}(\mathrm{p}, \gamma)^{21} \mathrm{Na}$. Upper solid line is for the capture to the $2.425 \mathrm{MeV}$ excited state of ${ }^{20} \mathrm{Ne}$ and lower solid line for the $0.332 \mathrm{MeV}$ excited state. Experimental data are from Ref. [100]. The dashed and dotted lines are theoretical results from Ref. [100] and Ref. 101], respectively 


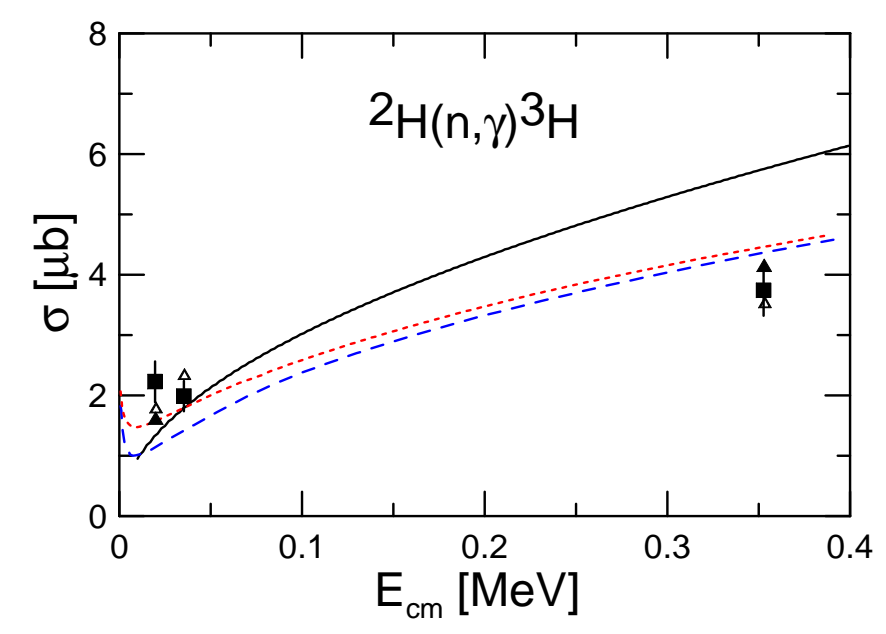

Figure 15: (Color online). Single-particle model calculation for ${ }^{2} \mathrm{H}(n, \gamma)^{3} \mathrm{H}$ (solid line). The experimental data are from Ref. [110]. The phenomenological results (parameter fit) from from Ref. [110] are shown by dashed and dotted lines. Also shown are microscopic calculations with (open trianges) and without (solid triangles) a three-body interaction.

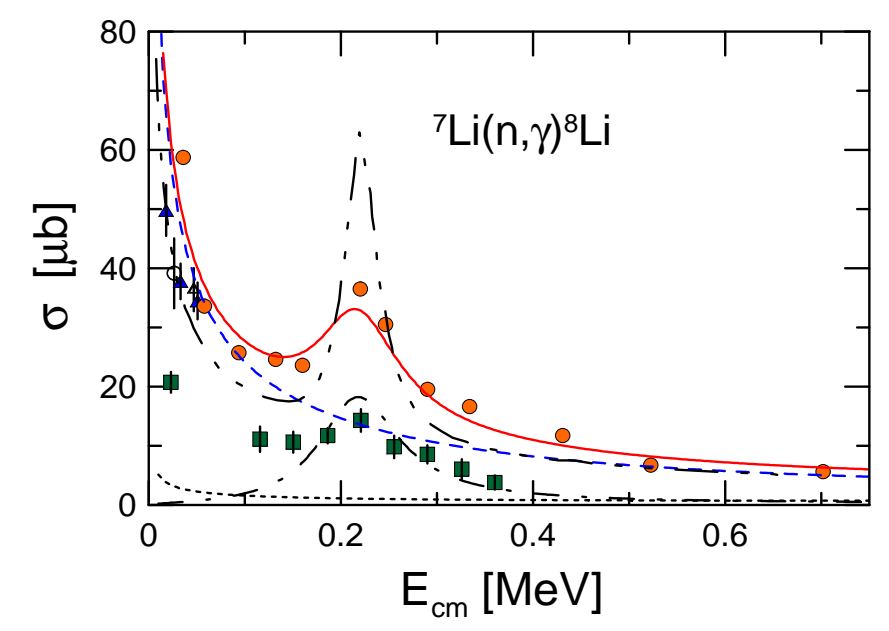

Figure 16: (Color online). Single-particle model calculation for the reaction ${ }^{7} \mathrm{Li}(\mathrm{n}, \gamma)^{8} \mathrm{Li}$. The dashed and dotted lines are for the capture to the ground state and first excited state, respectively. The dotted-dashed line is the calculated M1 resonance. The total cross section is shown as a solid line. The calculation result from Ref. 115 is shown as a dotted-dotted-dashed line. The experimental data are from refs. 115, 116, 117, 118, 119]. 


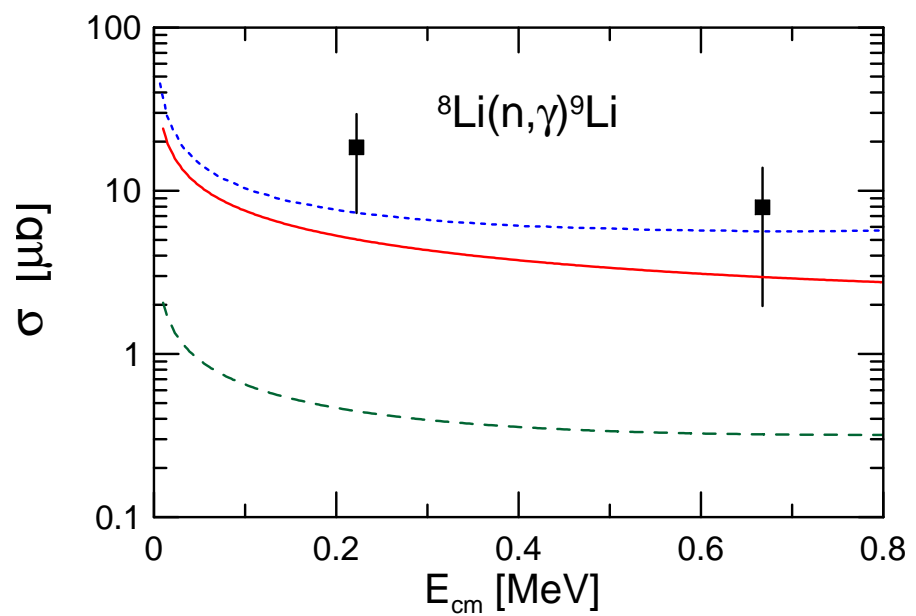

Figure 17: (Color online). Single-particle model calculation for ${ }^{8} \mathrm{Li}(n, \gamma){ }^{9} \mathrm{Li}$. The solid and the dashed lines are the calculations for the capture to the ground and the 1st excited states, respectively. The experimental data are from Ref. [127] using the Coulomb dissociation of ${ }^{9} \mathrm{Li}$ on $\mathrm{Pb}$ targets at $28.5 \mathrm{MeV} / \mathrm{A}$ beam energy. The dotted line is the calculation reported in Ref. [128] for the capture to the ground state.

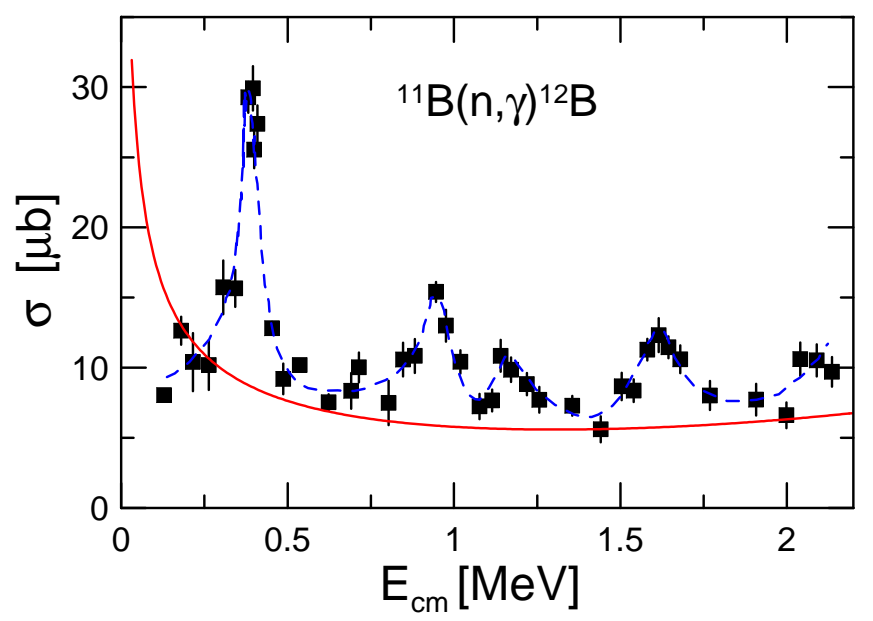

Figure 18: (Color online). Single-particle model calculation for the (non-resonant) capture reaction ${ }^{11} \mathrm{~B}(n, \gamma){ }^{12} \mathrm{~B}$ (solid line). The experimental data are from Ref. [131]. The dashed line is a sum of fitted Breit-Wigners superimposed to the non-resonant capture calculation, following Ref. [131]. 

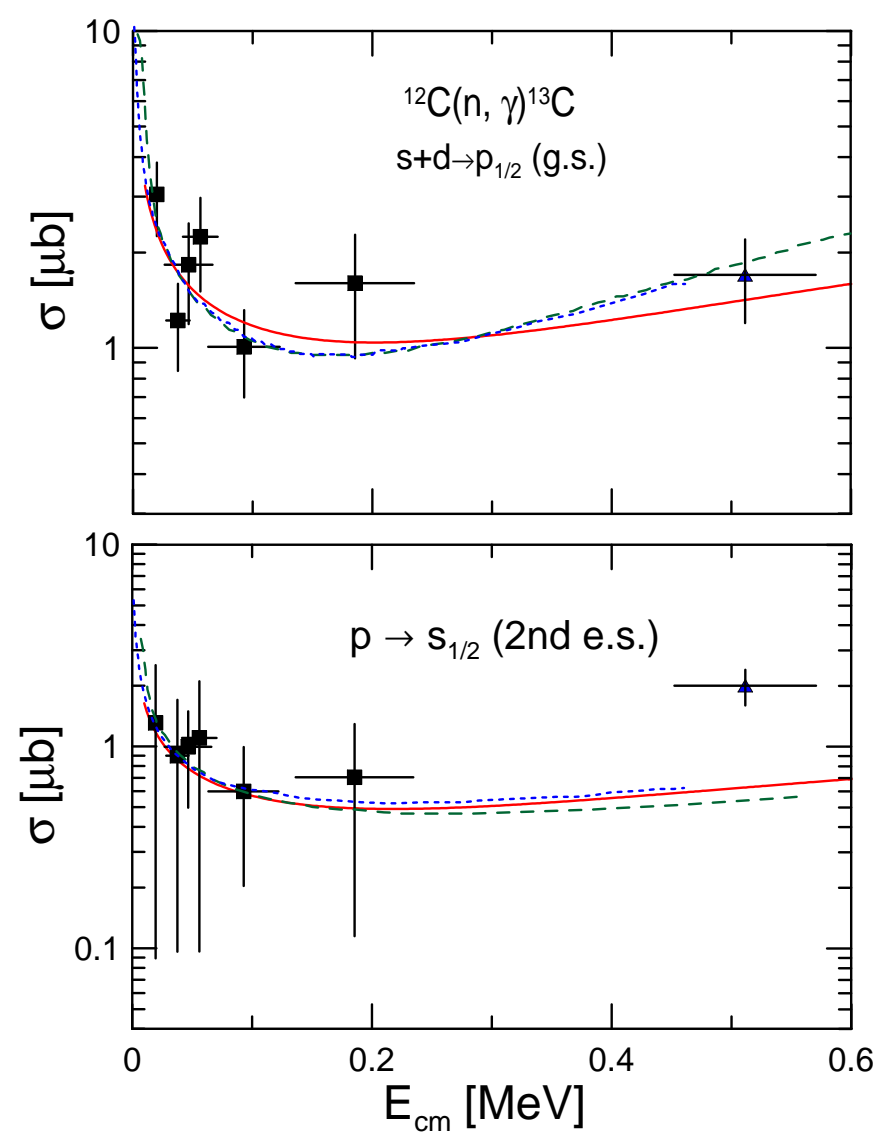

Figure 19: (Color online). Single-particle model calculation for ${ }^{12} \mathrm{C}(\mathrm{n}, \gamma){ }^{13} \mathrm{C}$ (solid line).The upper panel is for the capture to the ground state whereas the lower one is for capture to the 2nd excited state. The experimental data are from Ref. 134] (filled square) and Ref. [135] (filled triangle). The theoretical results from Ref. [135] and Ref. [136] are shown by the dashed and the dotted lines, respectively. 

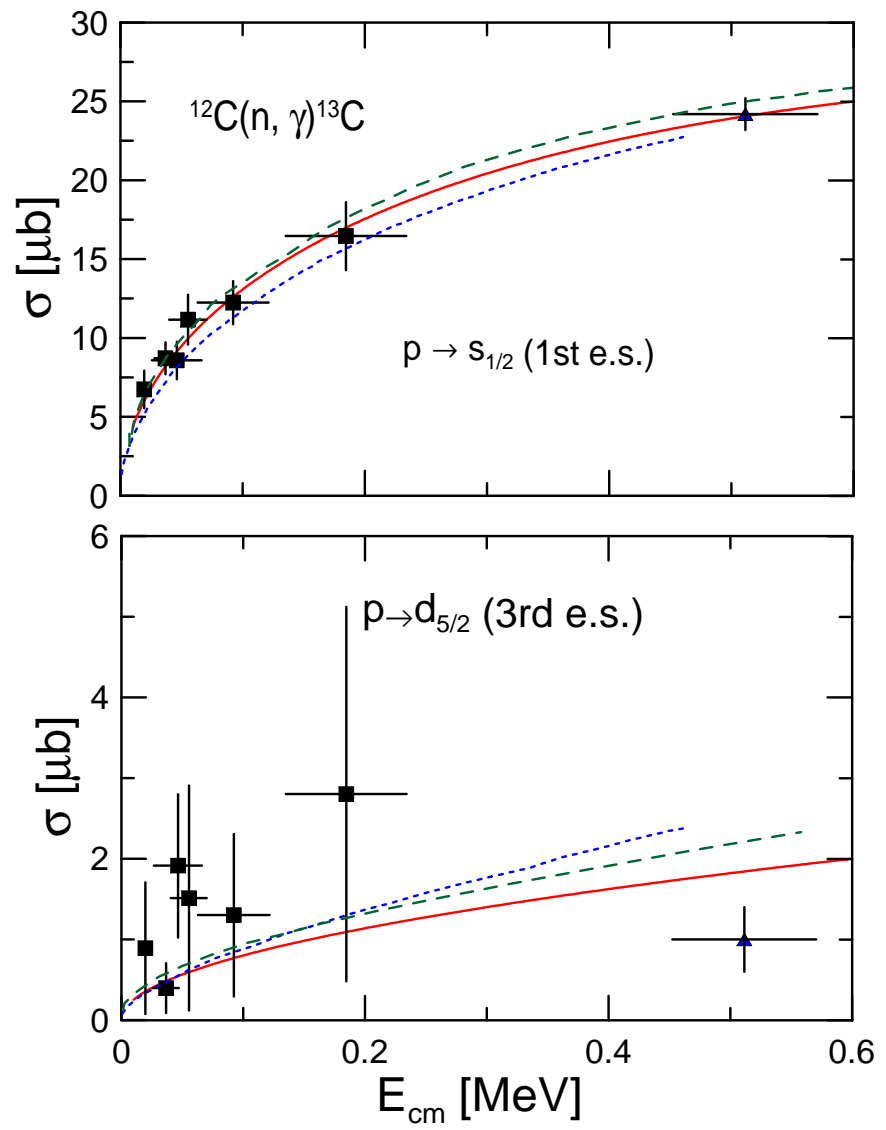

Figure 20: (Color online). The same as Fig. 19, but for the transitions to the 1st excited state (upper panel) and to the 3rd excited state (lower panel). 


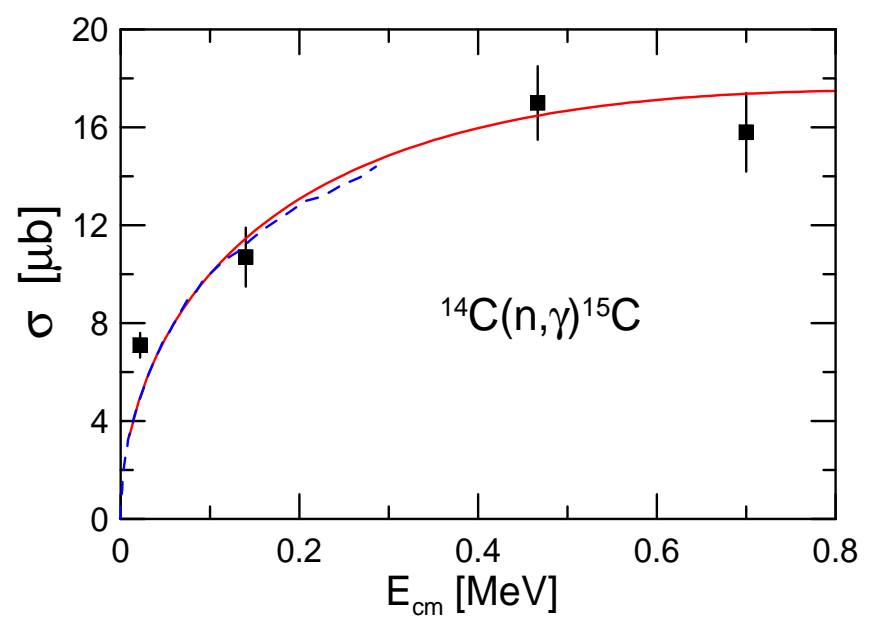

Figure 21: (Color online). Single-particle model calculation for the reaction ${ }^{14} \mathrm{C}(n, \gamma){ }^{15} \mathrm{C}$ (solid line). The experimental data are from Ref. [142]. The dashed line is the result from Ref. [143] using a similar potential model.

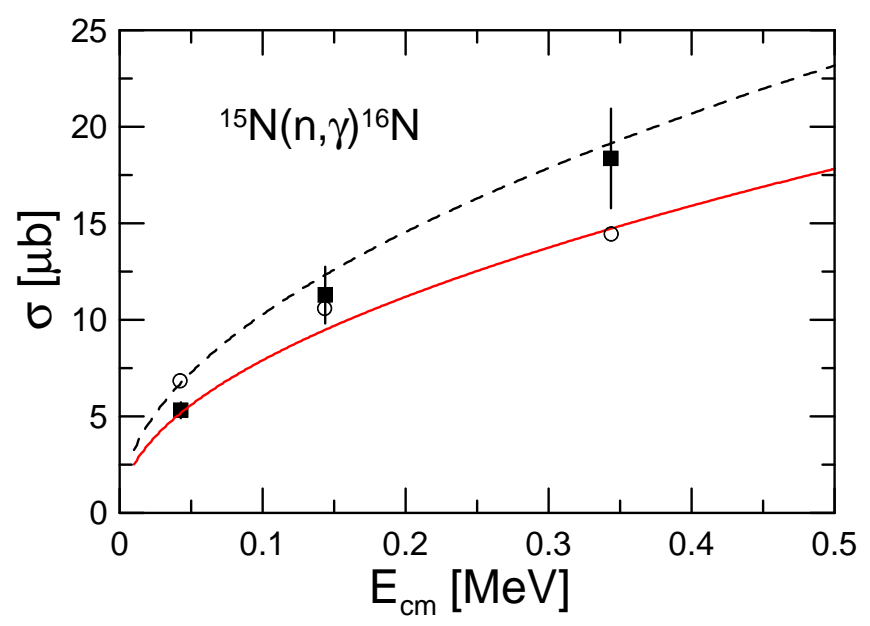

Figure 22: (Color online). Single-particle model calculation results for ${ }^{15} \mathrm{~N}(n, \gamma){ }^{16} \mathrm{~N}$ (solid line). The experimental data are from Ref. [145]. The non-resonant capture calculations of Ref. [145] is shown by open circles. Increasing the values of the spectroscopic values by $30 \%$ (compatible with the experimental errors) yields the dashed line. 

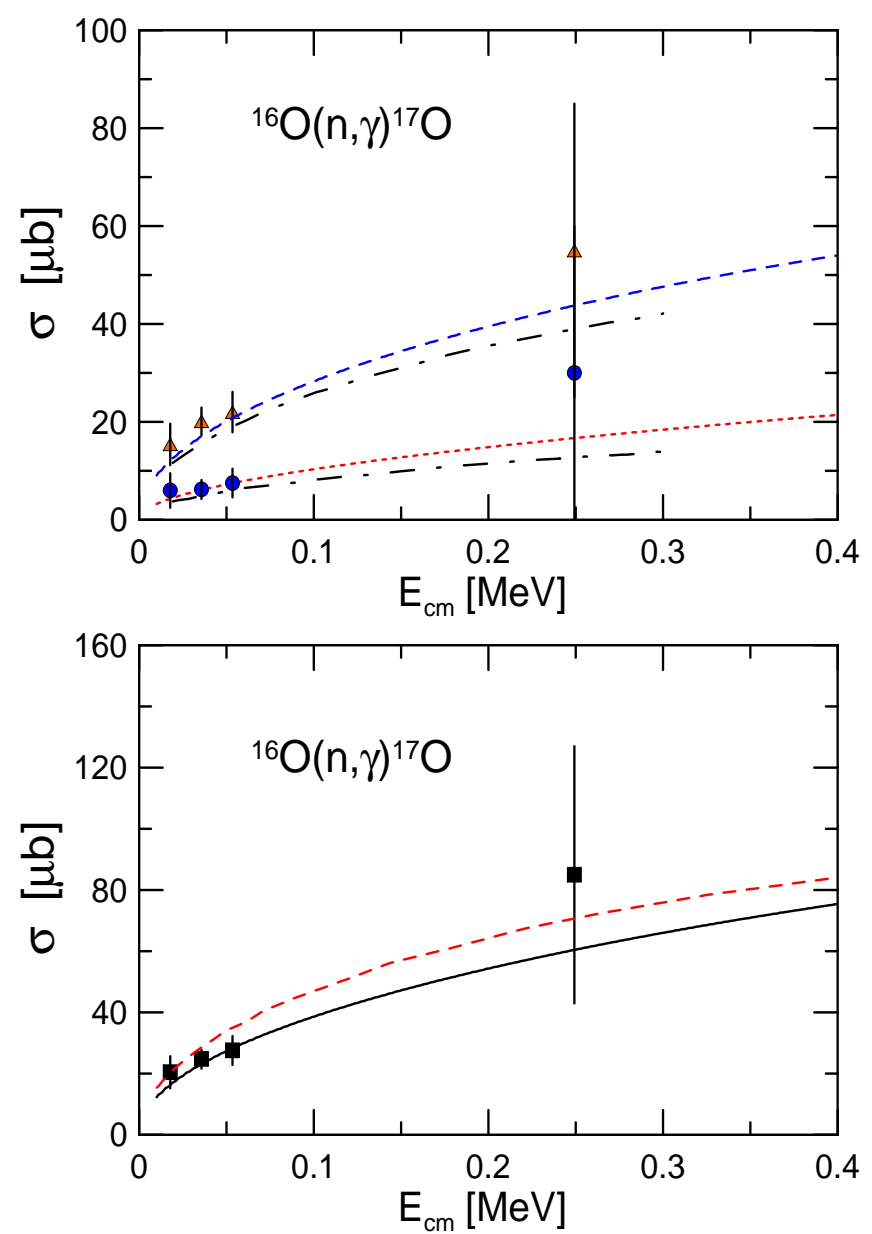

Figure 23: (Color online). Single-particle model calculation for reaction ${ }^{16} \mathrm{O}(n, \gamma){ }^{17} \mathrm{O}$ (solid lines). The experimental data are from Ref. [148]. Top panel: the capture to the ground state (dotted line, filled circles) and first excited state (dashed line, filled triangles) of ${ }^{17} \mathrm{O}$ are shown separately. The results of a microscopic multicluster model from Ref. [149] are shown by dotted-dashed lines for comparison. Bottom panel: the total cross section of ${ }^{18} \mathrm{O}(n, \gamma){ }^{19} \mathrm{O}$ (solid line). The result from Ref. [150] is shown as a dashed line. 


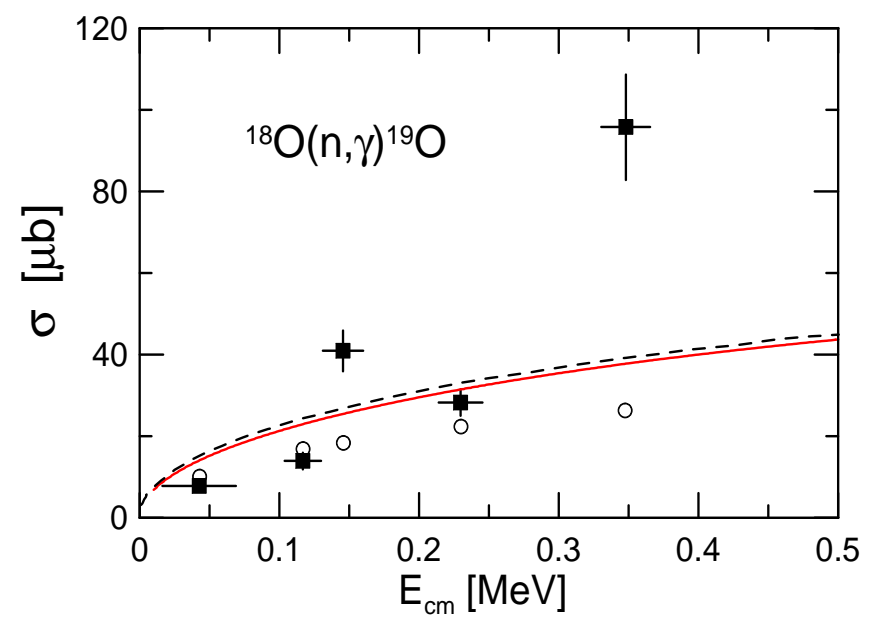

Figure 24: (Color online). Single-particle model calculation for the reaction of ${ }^{18} \mathrm{O}(n, \gamma){ }^{19} \mathrm{O}$ (solid line). The experimental data are from Ref. [151]. The non-resonant capture calculation from Ref. [151] and [147] are shown as open circles and dashed line, respectively.

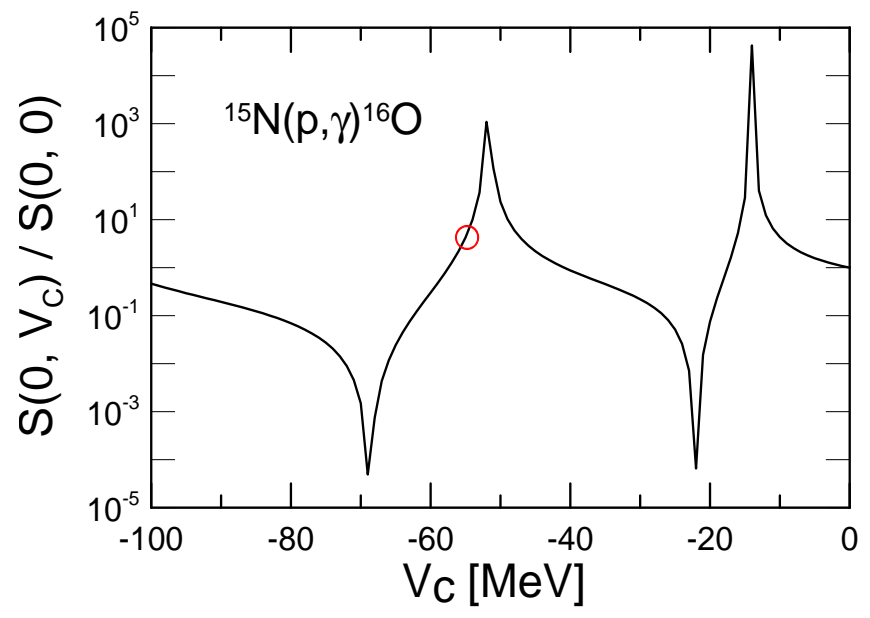

Figure 25: (Color online). Ratio between the S-factor at $E=0$ calculated with a potential depth $V_{c}$ and the S-factor calculated with a zero potential depth: $S\left(0, V_{c}\right) / S(0,0)$. The open circle corresponds to the value of $V_{c}$ used in the calculation presented in figure 12 . 


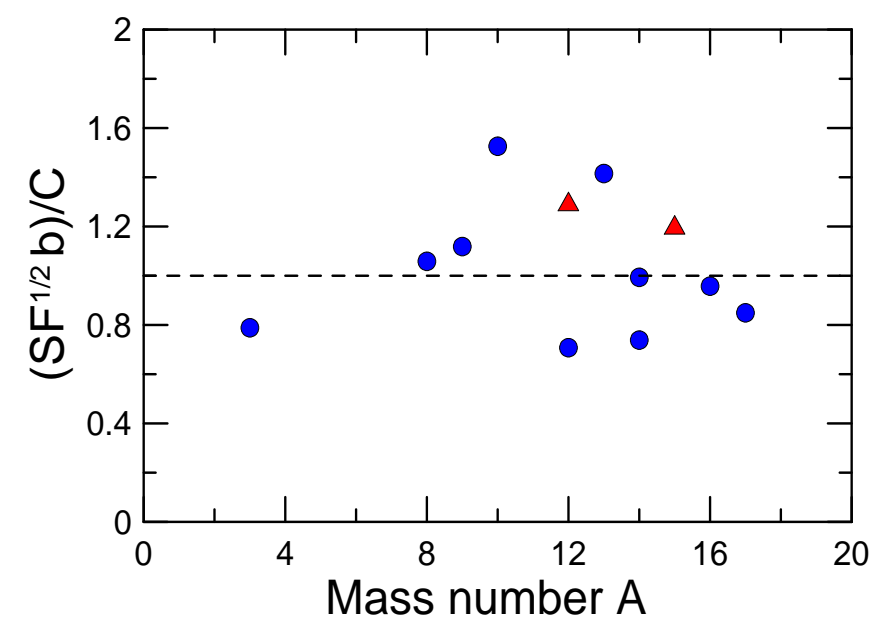

Figure 26: (Color online). Our ANCs $\left(\sqrt{(S F) b^{2}}\right)$ divided by the ANCs obtained from references mentioned in the text as function of the mass number $A$. The solid circles are for proton capture whereas the solid triangles are for neutron capture. The dashed line is equal to unity. 